\title{
Parallel parsing in a Gradient Symbolic Computation parser
}

\author{
Pyeong Whan Cho ${ }^{1}$, Matthew Goldrick ${ }^{2}$, and Paul Smolensky 3,4 \\ ${ }^{1}$ Department of Psychology, University of Michigan, Ann Arbor, MI \\ ${ }^{2}$ Department of Linguistics, Northwestern University, Evanston, IL \\ ${ }^{3}$ Department of Cognitive Science, Johns Hopkins University, Baltimore, MD \\ ${ }^{4}$ Microsoft Research, Redmond, WA
}

April 21, 2020

\begin{abstract}
In many cognitive domains, comprehenders construct structured, discrete representations of the environment. Because information is distributed over time, and partial information may not unambiguously identify a single representation, multiple possible structures must be maintained during incremental comprehension. How can the continuous-time, continuous-state neural cognitive system address these challenges? We propose a neural network approach, building on previous research in the Gradient Symbolic Computation framework in the domain of sentence processing. We introduce brick roles, a neurallyplausible, scalable distributed representation encoding binary tree structures. The appropriate structure is computed via an optimization process implementing a probabilistic context-free grammar. In the face of structural uncertainty encountered during incremental parsing, optimization yields conjunctive blends: states where multiple possible structures are simultaneously present (vs. disjunctive representations such as probabilistic mixtures). The degree of blending is controlled via a commitment parameter which drives local parsing decisions. We introduce a novel training algorithm for learning optimization parameters, and an improved policy for controlling commitment over a range of grammars. This provides a computational foundation for developing proposals integrating continuous and discrete aspects of sentence processing.
\end{abstract}

Keywords: Sentence processing, parsing, Gradient Symbolic Computation, neural networks

\section{Introduction}

Humans can rapidly understand speech, text, and sign as it unfolds. At the computational level of analysis (Marr, 1982), this ability can be analyzed as a series of belief updates on candidate meanings of a sentence in response to a sequence of language. Idealizing, such that each word is treated as a discrete input: for the $k$-th word $w_{k}$ of a sentence $w_{1} w_{2} \cdots w_{N}=w_{1: N}$, the language processing system rationally updates its belief, expressed as a probability distribution over meanings, from $P\left(M \mid w_{1:(k-1)}\right)$ to $P\left(M \mid w_{1: k}\right)$, where $M$ is 
a random variable over sentence interpretations or meanings; if we focus on the syntactic structure of a sentence as a core part of its interpretation, $M$ is a discrete random variable over tree structures ${ }^{1}$. This rational approach (e.g., Hale, 2001; Jurafsky, 1996; Levy, 2008) yields an optimal solution to a core computational problem in cognitive science: how the human language processing system deals with structural uncertainty naturally arising in online sentence comprehension.

This solution requires parallel parsing: simultaneously representing (either explicitly or implicitly) multiple structures, with different structures assigned different weights or probabilities. As we review below, evidence from human sentence comprehension experiments supports this analysis. For example, word reading times are correlated with the amount of uncertainty in the word sequence; this pattern of results is readily explained by parallel parsing (Boston et al., 2011).

While the computational structure of this solution is clear, it is not clear how the neural-cognitive system can achieve this goal. We need a theory of how the discrete aspects of linguistic computation, expressed in $P\left(M \mid w_{1: k}\right)$ at a discrete time point, can be related to the continuous aspects of the underlying process. In the present study, we assume that (A) sentence comprehension requires building a discrete hierarchical symbolic structure $^{2}$ and (B) the neural-cognitive system can be characterized as a continuous-time, continuous-state stochastic dynamical system. Under these assumptions, we pursue a potential neural-network solution to three computational questions.

(Q1) The mapping between a discrete set of symbolic structures and a continuous state space: how does the neural-cognitive system represent a discrete symbolic structure in its continuous state space?

(Q2) The mapping between a continuous state space and a set of probability distributions: how can the system represent degrees of belief over multiple symbolic structures in its continuous state space?

(Q3) The stability of the representation of multiple parses in the continuous state space: when the current input does not uniquely identify one symbolic structure, how can the system maintain multiple structures, without choosing one over the others, until disambiguating information arrives?

We propose an approach in the Gradient Symbolic Computation (GSC) framework (Smolensky et al., 2014). GSC builds on previous work proposing representational structures that allow neural networks to precisely realize symbolic structures (Smolensky, 1990; Smolensky and Legendre, 2006a,b) and provides an integrated account of the discrete and the continuous aspects of language processing - the outcome, and the transient dynamics, of linguistic computation. In particular, the intermediate activation states of the model are considered as conjunctive blends of multiple locally-coherent analyses, as opposed to disjunctive

\footnotetext{
${ }^{1}$ In most probabilistic models of sentence comprehension, only grammatical structures are considered as possible values of $M$. But this approach cannot explain that sometimes we fail to comprehend a sentence, apparently failing to build any grammatical structure - this type of failure is different from choosing a wrong but grammatical structure. Instead, we can consider a larger space $\mathscr{M}$, containing both grammatical and ungrammatical structures. For example, in constraint-satisfaction models (e.g., Legendre et al., 1990; MacDonald et al., 1994; Tabor and Hutchins, 2004), a global structure self-organizes or emerges from the cooperation and competition of local constraints and the structure may not be completely globally coherent.

${ }^{2}$ The vast majority of language scientists agree that the human language processing system computes hierarchical structures (Chomsky, 1957; but see Frank et al., 2012). Existing behavioral (Pickering and Ferreira, 2008) and neuroimaging studies (Ding et al., 2016; Nelson et al., 2017) provide converging evidence for hierarchical syntactic representations. In order for the sentence processing system to correctly apply knowledge over these compositional hierarchical representations, these representations must be approximately discrete (Smolensky et al., 2014).
} 
probabilistic mixtures of multiple globally-coherent analyses. A GSC model of incremental parsing (Cho et al., 2017) applied the GSC framework to online sentence comprehension, focusing on the third computational question: how to maintain uncertainty reliably across words. A unique aspect of this approach is viewing online sentence comprehension as an optimization process with optimal control of structural uncertainty. In particular, the parser uses a control parameter (called commitment strength) to gradually penalize blend states, thereby driving local parsing decisions. Under optimal control of commitment strength (a commitment policy), Cho et al. (2017) showed that the model was able to successfully maintain structural uncertainty. Under suboptimal commitment policies, the model made types of parsing errors that are observed in human behavioral experiments. In that work, the key problems in maintaining structural ambiguity posed by Q3 were reduced to their purest, minimal form, instantiated in two-word sentences. It was not clear whether and how the model could be extended to process more complex examples.

In the present study, our main goal is to propose a (continuous) neurally plausible, scalable solution to the (discrete) computational problems raised by temporary ambiguity. We address this by extending the GSC parser in three key ways:

1. We propose a novel, neurally plausible, and scalable representation scheme for binary tree structures.

2. We propose a heuristic training algorithm that allows a GSC model to approximate probabilistic context-free grammars.

3. We propose an improved commitment policy scheme that can successfully process a wider range of context-free grammars.

Although our ultimate interest is modeling human sentence processing, the technical aspects of this solution demand extended exposition, and are the subject of this paper; work assessing the empirical validity of the approach for explaining human on-line sentence processing behavior will be presented in subsequent papers.

In the following section, we introduce and discuss the three core computational problems arising when trying to bridge between the discrete and the continuous aspects of incremental language processing. In Section 3, we introduce the Gradient Symbolic Computation incremental parser and show how to initialize and train the model to approximate any given probabilistic context-free grammar (PCFG). In Section 4, the model is tested on a few simplified PCFGs, each of which generates psycholinguistically interesting English-like constructions. In Section 5, we evaluate the technical aspects our model presented here, and discuss future directions.

\section{Background}

Language processing is a core component of human cognition. Neurotypical individuals can easily produce and comprehend sentences of their native language in real time. However, online sentence comprehension can be computationally challenging. Suppose you listen to the following sentences: "The horse raced past the barn fell" (Bever, 1970) and "The coach smiled at the player tossed a frisbee by the team" (Tabor et al., 
"The dog chases the cat."

$\mathrm{S}(\mathrm{NP}(\operatorname{Det}($ the $), \mathrm{N}(\operatorname{dog})), \mathrm{VP}(\mathrm{V}($ chases $), \mathrm{NP}($ Det(the $), \mathrm{N}($ cat $))))$

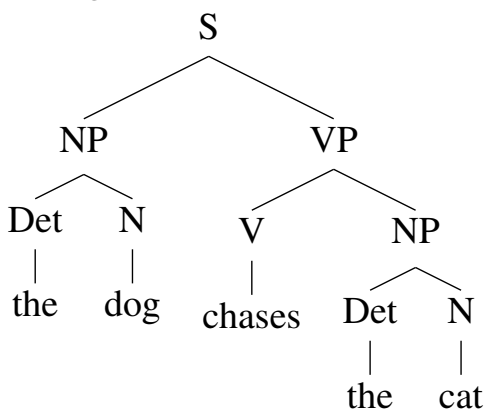

Figure 1: Simple parse tree, showing the combinatorial structure of the component words, with accompanying bracket notation. 'S': Sentence; 'NP': Noun Phrase; 'VP': Verb Phrase; 'Det': Determiner; 'N': Noun; 'V': Verb

2004). Native speakers of English have trouble understanding the underlined regions, although both sentences are completely grammatical. On the other hand, some sentences sound good but are ungrammatical. An example is "The patient who the nurse who the clinic had hired met Jack" where a verb, the subject of which can be either "nurse" or "patient", is missing (Frazier, 1985; see also Gibson and Thomas, 1999). These cases provide researchers with good opportunities to investigate how the human language processing system works and what limitations it has.

A diverse set of factors contribute to the computational challenges arising in the above examples. One important source is the combinatoriality of language. Language is a discrete combinatorial system (Hockett, 1960; Pinker, 1994). At the sentence level, words can combine with other words in many different ways to yield different meanings. Combinatoriality makes human language highly expressive with a limited number of words. The benefit comes with a cost. Combinatoriality creates one-to-many mappings from each individual word to many sentences and their meanings; given a sentence, each individual word is locally structurally ambiguous with respect to its contribution to the meaning of the sentence - although the whole sentence, the complete combination of words, is not typically ambiguous. This local ambiguity poses a serious challenge when words are perceived sequentially. Given limitations in human working memory (Cowan, 2001; Miller, 1956), holding all individual words in a memory buffer until the last word of a sentence comes in and then analyzing the meaning of the sentence is not feasible. The language processing system must compute partial interpretations and update them incrementally with every new word input (Christiansen and Chater, 2016). The core computational problem in online sentence comprehension is therefore how to maintain temporary ambiguity or structural uncertainty while incrementally processing linguistic input so that the processing system can successfully recover the intended meaning.

Rational models (e.g., Hale, 2001; Jurafsky, 1996; Levy, 2008) provide a way of solving these problems. Roughly speaking, the language processing system builds an internal model of the linguistic environment which can be expressed as a probability distribution over different interpretations, for example, in the form of complete parse trees, which specify the combinatorial structure of a full utterance (see Figure 1 for an illustration; note that the bracket notation will be utilized in the text below). The probability of each inter- 
pretation is taken as a belief strength. Whenever the language processing system receives a new word input, it updates its beliefs about different interpretations based on the language model, which can be approximated by probabilistic context-free grammars or neural network models (e.g., Simple Recurrent Network models [Elman, 1990] or Long Short-Term Memory models [Hochreiter and Schmidhuber, 1997]); n.b. in most neural language models, the representation of multiple analysis possibilities is not explicit.

Rational models predict the processing difficulty of each word in a sentence based on the change in the probability distribution induced by that word. More specifically, processing difficulty is explained in terms of information-theoretic complexity metrics, either surprisal (Hale, 2001; Levy, 2008) or entropy reduction (Hale, 2003) (for a review, see Hale, 2016). Surprisal effects have been reported in multiple domains, including reading time data (Boston et al., 2008, 2011; Demberg and Keller, 2008; Lowder et al., 2018; Roark et al., 2009; Smith and Levy, 2013), pupil sizes (Frank and Thompson, 2012), event-related potentials (Frank et al., 2015), and fMRI activations (Henderson et al., 2016; Willems et al., 2016). The sensitivity of language processing mechanisms to the amount of uncertainty can be accounted for by parallel parsers that directly represent degrees of uncertainty via the maintenance of multiple analyses consistent with the current input.

It should be noted that human comprehenders often commit substantial processing resources to the most likely parse (e.g., that the horse raced rather than fell in "The horse raced past the barn fell" — the 'garden path' effect [Bever, 1970; Frazier and Rayner, 1982]). Parallel processing models can account for such limitation by assuming limitations of computational resources for maintaining parses (e.g., Jurafsky, 1996). Further supporting a parallel parsing analysis of these phenomena, when comprehenders must reanalyze a difficult-to-process word string, the cost of reanalysis is graded (Ferreira and Henderson, 1991; Tabor and Hutchins, 2004) and related to how expected the alternative analysis is (Van Schijndel and Linzen, 2018), suggesting that the alternative analyses are not completely suppressed.

Additional evidence that comprehenders consider multiple parses comes from research showing that listeners entertain context-inappropriate interpretations (e.g., that the player tossed a frisbee rather than was tossed a frisbee in "The coach smiled at the player tossed a frisbee by the team") (Konieczny, 2005; Tabor et al., 2004). This suggests that comprehenders can activate (partial) parses that are incompatible with parses that are already being pursued.

Finally, inspired by Traxler et al. (1998)'s study in which people read globally ambiguous sentences more quickly than unambiguous sentences, Dillon et al. (2017) collected and analyzed speeded acceptability judgment of globally ambiguous (e.g., "Armand spotted the cousin of the painter who knits" where either the cousin or the painter knits) or unambiguous sentences ("Armand spotted the cousins of the painter who knits", where the painter knits [low attachment], or "Armand spotted the cousin of the painters who knits", where the cousin knits [high attachment]). Their analyses reveal that the distribution of acceptability ratings for ambiguous sentences is jointly conditioned by multiple parses, consistent with parallel parsing.

Given the array of evidence supporting parallel parsing, a question naturally follows: How does the human brain simultaneously maintain multiple, discrete symbolic interpretations simultaneously? 


\subsection{Tensor product representations of discrete symbolic structures}

Any symbolic structure can be viewed as a set of symbols bound to different structural roles (Newell, 1980; Smolensky, 1990). For example, a tree structure VP(V, NP) (which can be the syntactic analysis of "hit the-ball") can be described as $\{\mathrm{V} /$ leftChild, VP/parent, NP/rightChild $\}$ where the symbols VP, V, and NP are respectively bound to the structural roles (e.g., node positions in a tree) parent, leftChild, and rightChild. (n.b. In this paper, we consider grammars over lexical categories like V[erb], N[oun] and do not implement lexical rules such as $\mathrm{V} \rightarrow$ hit; for justification, see Hale and Smolensky, 2006. For brevity, we sometimes refer to these categories as "words".) A fundamental representational question is how the neural-cognitive system encodes filler/role (or value/variable) bindings, which themselves can bind to other roles recursively.

Smolensky (1990) (see also Dolan, 1989; Dolan and Smolensky, 1989; Smolensky, 2006; Smolensky and Tesar, 2006) proposed a general solution to this problem, tensor product variable binding, in which the binding of a filler symbol to a role is represented as the tensor product (or generalized outer product) of the filler representation vector and the role representation vector; in general, these vectors constitute distributed representations. ${ }^{3}$ Encoding structural roles as vectors is a key innovation of this approach. As an example, $\mathrm{VP} /$ parent $\equiv \mathbf{f}_{\mathrm{VP}} \otimes \mathbf{r}_{\text {parent }}$, where $\mathbf{f}_{\mathrm{VP}}$ and $\mathbf{r}_{\text {parent }}$ are respectively the vector encodings of the filler, VP, and the role, parent. Then, a symbolic structure is represented as the superposition (or vector sum) of its constituent bindings: $\mathrm{VP}(\mathrm{V}, \mathrm{NP}) \equiv \mathbf{f}_{\mathrm{VP}} \otimes \mathbf{r}_{\text {parent }}+\mathbf{f}_{\mathrm{V}} \otimes \mathbf{r}_{\text {leftChild }}+\mathbf{f}_{\mathrm{NP}} \otimes \mathbf{r}_{\text {rightChild. }}$. Compositionality naturally follows from this representational scheme. Assuming the linear independence of binding vectors, this approach provides a general solution to the binding problem, free from the superposition catastrophe (in which the distinction between roles encoded in two vectors is lost when they are combined; von der Malsburg, 1999). For example, $\mathbf{f}_{\mathrm{V}} \otimes \mathbf{r}_{\text {leftChild }}+\mathbf{f}_{\mathrm{NP}} \otimes \mathbf{r}_{\text {rightChild }} \neq \mathbf{f}_{\mathrm{NP}} \otimes \mathbf{r}_{\text {leftChild }}+\mathbf{f}_{\mathrm{V}} \otimes \mathbf{r}_{\text {rightChild }}$ (whereas $\mathbf{f}_{\mathrm{V}}+\mathbf{f}_{\mathrm{NP}}=\mathbf{f}_{\mathrm{NP}}+\mathbf{f}_{\mathrm{V}}$ ).

By recursive applications of the tensor product binding operator, this approach can, in principle, map any symbolic structure to a vector in a continuous state space. For example, the tree structure VP(V, NP) can be bound to a structural role 'rightChild' in a larger tree $\mathrm{S}(\mathrm{NP}, \mathrm{VP}(\mathrm{V}, \mathrm{NP})$ ). Note that the tensor product operation increases the dimensionality of the representation space (e.g., if fillers [which themselves can be the superposition of filler/role bindings] are represented in $\mathbb{R}^{4}$ and roles are represented in $\mathbb{R}^{2}$, then their bindings are represented in $\mathbb{R}^{8}$ ). In practice, the representation space is assumed to have a finite dimensionality so there is an upper bound on embeddings up to which the system can deal with perfectly (see Gayler, 2003; Plate, 1995 for an alternative approach to binding utilizing fixed-dimensionality representations).

Although not widely recognized, many classical connectionist models of language and cognition instantiate the tensor-product variable-binding representation (Smolensky and Tesar, 2006). For example, the Interactive Activation and Competition model of visual word recognition (McClelland and Rumelhart, 1981; Rumelhart and McClelland, 1982) assigns a group of units (e.g., letter identity) to a particular role (e.g., letter position in a letter string). The TRACE model (McClelland and Elman, 1986) has multiple copies of units each of which can be viewed as representing a binding of a symbol (e.g., a phoneme) to a structural role (a position in time). ${ }^{4}$

\footnotetext{
${ }^{3}$ The tensor product of two vectors $\mathbf{f}$ and $\mathbf{r}$ can be represented by a matrix $\mathbf{T}$ in which the $i, j$ element is the product $T_{i j}=f_{i} r_{j}$

${ }^{4}$ The popular framework of convolutional neural networks (LeCun et al., 1998) also instantiates tensor product variable binding, where each unit in a filter represents a what/where (or value/variable) binding (Edelman, 2002).
} 
Related, and more specialized to sentence processing, are dynamic network models of sentence processing (e.g., Smith et al., 2018; Stevenson, 1994; Tabor and Hutchins, 2004; Vosse and Kempen, 2000, 2009) which represent tree structures explicitly and compute the structured representation gradually across multiple time steps, sometimes with additional symbolic operations (e.g., Stevenson, 1994). This class of models assigns a group of units (each of which represents a linguistic feature [e.g., singular vs. plural]) to represent a symbol bound to a node in a tree or, more generally, in a graph. ${ }^{5}$

\subsection{Structural ambiguity and the conjunctive blend of locally-coherent symbolic substruc- tures}

Although tensor product variable binding is a neurally plausible solution to the binding problem in the representation of a single symbolic structure, representing multiple structures simultaneously seems to create a superposition catastrophe. Suppose that there are two parse trees (n.b. square brackets are used to indicate distinct types of syntactic elements; e.g., a plural vs. singular noun phrase): S[1](NP[1], VP[1]) and $\mathrm{S}[2](\mathrm{NP}[2], \mathrm{VP}[2])$ consistent with a partial sentence input and there are two ungrammatical trees $\mathrm{S}[1](\mathrm{NP}[1], \mathrm{VP}[2])$ and $\mathrm{S}[2](\mathrm{NP}[2], \mathrm{VP}[1])$ inconsistent with the input. For simplicity, let us assume each interpretation is equally likely. Since each symbolic structure is represented by the superposition of its constituents, the superposition of the two grammatical structures cannot be distinguished from the superposition of the two ungrammatical structures. 0.5 S[1](NP[1], VP[1]) + 0.5 S[2](NP[2], VP[2]) $\equiv 0.5$ $(\mathrm{S}[1] /$ parent $+\mathrm{NP}[1] /$ leftChild $+\mathrm{VP}[1] /$ rightChild $)+0.5(\mathrm{~S}[2] /$ parent $+\mathrm{NP}[2] /$ leftChild $+\mathrm{VP}[2[/$ leftChild $)$ $=0.5(\mathrm{~S}[1] /$ parent $+\mathrm{NP}[1] /$ leftChild $+\mathrm{VP}[2] /$ rightChild $)+0.5(\mathrm{~S}[2] /$ parent $+\mathrm{NP}[2] /$ leftChild $+\mathrm{VP}[1] /$ leftChild $)$ $\equiv 0.5 \mathrm{~S}[1](\mathrm{NP}[1], \mathrm{VP}[2])+0.5 \mathrm{~S}[2](\mathrm{NP}[2], \mathrm{VP}[1])$.

In dynamic neural network models, a general solution to this problem is (1) to introduce into the processing dynamics constraints that specify which tensor product bindings can co-occur with which other bindings, and (2) to use the resulting processing dynamics to unblend the ambiguous blend representation (e.g., Rasmussen and Schuler, 2018; Tabor and Hutchins, 2004; Vosse and Kempen, 2000). For example, a processing unit representing S1/parent will have a bidirectional excitatory connection with a processing unit representing NP1/leftChild but not with another processing unit representing NP2/leftChild. In this case, as the activation of S1/parent increases, the activation of NP1/leftChild increases but the activation of NP2/leftChild does not.

We emphasize that blend states must be distinguished from probabilistic mixtures of multiple globallycoherent interpretations. For example, consider the activation state blend $0.5 \mathrm{~S}[1](\mathrm{NP}[1], \mathrm{VP}[1])+0.5$ $\mathrm{S}[2](\mathrm{NP}[2], \mathrm{VP}[2])$. In this state, neither NP[1] nor NP[2] occupies the leftChild role alone. This role is occupied by a conjunctive blend of NP[1] and NP[2] (technically, a conjunctive blend of all filler symbols, most with activity 0$).{ }^{6}$ The conjunctive blend has the properties of both NP[1] and NP[2], which can resonate with external input supporting either analysis. If no disambiguating information is available, under the stochastic processing dynamics the conjunctive blend will be unblended, evolving toward either pure

\footnotetext{
${ }^{5}$ Other models (MacDonald et al., 1994; McRae et al., 1998) take similar approaches to representing linguistic objects but without trying to represent complete parse trees directly.

${ }^{6}$ From a different but equivalent perspective, symbol NP[1] is bound to a conjunctive blend of leftChild and rightChild, the latter with activity 0 .
} 
$\mathrm{NP}[1]$ or pure NP[2], each with some probability (although dynamic unblending may not be complete). This process is different from sampling either the pure NP[1] or pure NP[2] in probabilistic models, and certainly different from sampling the pure global structure S[1](NP[1], VP[1]) or pure S[2](NP[2], VP[2]) — with zero probability of sampling S[1](NP[1], VP[2]).

\subsection{Dynamic control of stability of the conjunctive blend state}

Consider what the neural-cognitive representation will look like after processing the first $k$ words of a sentence but before processing the $(k+1)$-th word. For the purposes of discussion, consider two sentences 'the player [that was] tossed the frisbee eats' vs. 'the player tossed the frisbee'. Across these sentences, 'tossed the frisbee' has distinct grammatical analyses; it is either part of a relative clause (e.g., RC(Verb, NP)) or it forms a verb phrase ((VP(Verb, NP)). After processing this portion of the sentence, the neural-cognitive state can take one of three forms.

(H1) Conjunctive blend: The intermediate state is the blend of multiple, partially active symbols at different positions in a parse tree. Consider the parse fragment ([0.7 VP + 0.5 RC](1.0 Verb, 1.0 NP) where the numeric values represent the activation strengths of symbols at different positions in a parse tree. Both VP and RC are partially active. Material to the right can be either an empty symbol '_, or a conjunctive blend (e.g., $0.7 @+0.5 \mathrm{VP}$, where @ marks end of sentence). The latter constitutes expectation of future inputs (i.e., that the sentence will end or a verb phrase will follow).

(H2) Single analysis: The intermediate state represents a fully active, partial parse tree: either RC(Verb, NP) or VP(Verb, NP). The parser may choose the analysis either randomly (e.g., van Gompel et al., 2001) or based on heuristics (e.g., minimal attachment and late closure; Frazier, 1987). We note that this does not necessarily mean that the state represents a single complete parse tree.

(H3) Single analysis with underspecification: The parser delays a decision between competing interpretations until disambiguating information becomes available. Thus, the state after processing the prefix corresponds to _(Verb, NP) where '_' represents an empty node. ${ }^{7}$

We note that all three cases can result from brief activations of multiple interpretations as each word is processed. The question here is whether the system chooses a single interpretation or maintains multiple possibilities before the following word (or more generally, disambiguating information) comes in. ${ }^{8}$ In the

\footnotetext{
${ }^{7}$ The same solution can be applied to a feature-level representation. Suppose that a symbol $\mathrm{N}$ is represented as a feature list where the model uses two units to represent the number of the noun, one for singular and one for plural. When no information on the number of the noun is available from the partial sentence input, the values of two number units can stay at $(0,0)$ rather than taking a blend of multiple possibilities $(0.7,0.3)$. We take the former state as the underspecification of the number feature, which should be distinguished from choosing a preferred option (e.g., 'singular'; (1,0)). An alternative is to use a single unit to encode the feature '+ + plural'. In this case, a low activation value of the unit is interpreted as suggesting singular or 'an intermediate value such as 0.5 as the blend of singular and plural, not as the underspecification of the number feature.

${ }^{8}$ With regard to the distinction between serial and parallel parsing, the unrestricted race model (van Gompel et al., 2001) does consider multiple interpretation possibilities with each word input but randomly chooses one over the others before processing the next word. Similarly, the cue-based retrieval model (Lewis and Vasishth, 2005) considers multiple analyses for every new word input but chooses one before moving to the next word. Because theses models maintain only one single analysis across words, they will be treated as serial parsing models.
} 
context of dynamical neural network models, what distinguishes among the three hypotheses $\mathrm{H} 1-\mathrm{H} 3$ is whether the blend state discussed in the previous section is dynamically stable or not. If it is unstable, the system will eventually move away from the blend state and move toward a state corresponding to either $\mathrm{H} 2$ or $\mathrm{H} 3$. We will refer to a system that has the ability of developing a stable blend state as a truly parallel parser in the sense that multiple interpretations are maintained across words.

Most dynamical network models of sentence processing have the ability to transiently activate multiple local parse structures but do not have the capability to maintain multiple interpretations reliably. This is because disambiguation in those models relies heavily on competitive dynamics introduced by static inhibitory connections among competing interpretations (which help implement attractors for states corresponding to a single analysis). In such models, a small amount of perturbation (introduced either by model-intrinsic noise or by environmental noisy input) can easily move the system away from the blend state and towards a state representing a single analysis (yielding $\mathrm{H} 2$ or $\mathrm{H} 3$ ). In order to pursue the possibility of stable blend states (H1), we require a different mechanism that yields distinct dynamics.

The Gradient Symbolic Computation (GSC) model of sentence processing (Cho et al., 2018, 2017), presented next, is a rare exception that takes the solution of the first kind, maintaining structural uncertainty in a stable blend state. (For a related approach that posits quasi-stationary intermediate brain states, see beim Graben and Hutt, 2015; Hutt and beim Graben, 2017.) The GSC model takes the tensor-product variablebinding approach to explicitly represent discrete symbolic structures across multiple processing units in a distributed way. The model is a continuous-time, continuous-state, stochastic dynamical system that dynamically unblends the conjunctive representation of multiple interpretations, avoiding the superposition catastrophe. Unlike other models, the model has the ability to change the stability of blend activation states dynamically, in response to external word input and an internal control force called commitment strength. We will argue that the GSC model provides a neurally-plausible solution to all three representational challenges Q1-Q3 discussed in the Introduction. In the present work, we extend the GSC approach of Cho et al. $(2018,2017)$ by introducing numerous technical innovations which enable the model to cope with more computationally complex cases.

\section{Gradient Symbolic Computation parser}

A Gradient Symbolic Computation (GSC) model (Smolensky et al., 2014) is a continuous-time, continuousstate stochastic dynamical system in which the (activation) state changes, on average, in the direction of the gradient of the Harmony function that maps every state to a real number (Harmony) quantifying goodness of the state. (The Harmony of an activation state can be viewed as the negative energy of the state in energy-based models such as the continuous Hopfield network [Hopfield, 1984].) Building on the Harmonic Grammar formalism (Legendre et al., 1990; Smolensky and Legendre, 2006a,b) — which provides a way of implementing a collection of linguistic constraints into a (Grammar) Harmony function - GSC introduces processing dynamics in which the system starts from a blend state, passes through stable blend states, and approaches (without necessarily reaching) a 'pure' state (called a grid point) representing a single discrete symbolic structure that best satisfies the linguistic constraints of the Harmonic Grammar. This is achieved 
by introducing a general cognitive constraint that only one filler can occupy a structural role, violated by bona fide blend states. The relative contribution of this constraint is gradually increased over the course of processing.

Because the GSC framework is relatively new, in the following, we review previously reported components of the GSC model (Cho et al., 2018, 2017; Smolensky et al., 2014) as well as presenting our new contributions: a new representation scheme (Section 3.1.1), a novel commitment policy scheme (Section 3.5.2), and a heuristic training algorithm (Section 3.7); these address questions Q1, Q3, and Q2 of the Introduction, respectively.

\subsection{Vector representation of binary-branching tree structures}

Following Hale and Smolensky (2006), the previous GSC parser (Cho et al., 2017) used the span role system to represent tree structures in a vector space. A span role $(i, j)$ binds to either a terminal symbol spanning from position $i$ to position $j$ (if $j=i+1$ ) or a non-terminal symbol which has a child bound to role $(i, k, j)$. A span role $(i, k, j)$ binds to a non-terminal symbol that has a constituent spanning from position $i$ to position $k$ as its left child and a constituent spanning from position $k$ to $j$ as its right child. For example, consider 'the dog eats'; this has a structure $\mathrm{S}(\mathrm{NP}($ Det, $\mathrm{N}), \mathrm{V})$. Under the span role system, the structure can be described as $\operatorname{Det} /(0,1), \mathrm{N} /(1,2), \mathrm{V} /(2,3), \mathrm{NP}[1] /(0,1,2), \mathrm{NP} /(0,2), \mathrm{S}[1] /(0,2,3), \mathrm{S} /(0,3)$; the motivation underlying the bracketed symbols will be explained in the next section.

We suspect that GSC models adopting the span role system are not easily scalable for the following reasons. First, the number of span roles required to represent all possible binary-branching tree structures increases very quickly with sentence length: for a given maximum sentence length $N_{\max }$, in total $N_{\max }\left(N_{\max }+1\right)\left(N_{\max }+2\right) / 6$ roles are required. Second, as the maximum sentence length increases, more binding pairs (and between-binding interactions) must be considered in order to implement a given grammatical constraint. For example, suppose a terminal symbol A can be a grammatical left child of a branching non-terminal symbol X[1]. For symbol A bound to role $(i, i+1)$, its parent X[1] can bind to any of these span roles $(i, i+1, j)\left(j \in\left\{i+2, \cdots, N_{\max }\right\}\right)$, as A's sister constituent can span up to position $N_{\max }$. A GSC model needs to be constructed to allow the interaction between binding $\mathrm{A} /(i, i+1)$ and every binding $\mathrm{X}[1] /(i, i+1, j)$, which makes processing dynamics highly complex. In our preliminary investigations, we observed that GSC models adopting the span role system did not scale up well.

Inspired by Cho et al. (2014), we propose a new role system for the GSC parser, in which the number of roles required to represent all possible binary trees increases much more slowly with sentence length: $N_{\max }\left(N_{\max }+1\right) / 2$, for strings with length up to $N_{\max }$. In addition, the number of bindings that a binding interacts with remains constant regardless of sentence length, greatly simplifying the system's dynamics.

\subsubsection{Tree representation with brick roles}

To represent binary-branching trees, we introduce brick roles, structural roles corresponding to different locations in a stack of linearly ordered sequences (see Figure 2a). A brick role $(l, p)$ denotes the $p$-th position (from left to right) in a sequence of bricks in the $l$-th layer (from bottom to top). Brick role $(l, p)$ 


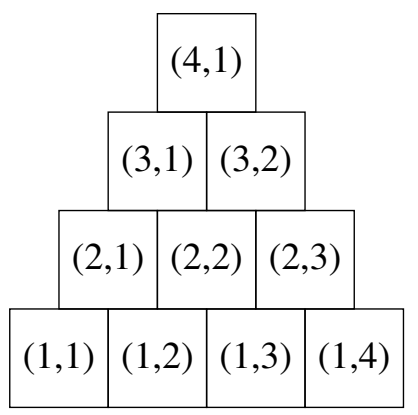

(a) Brick roles

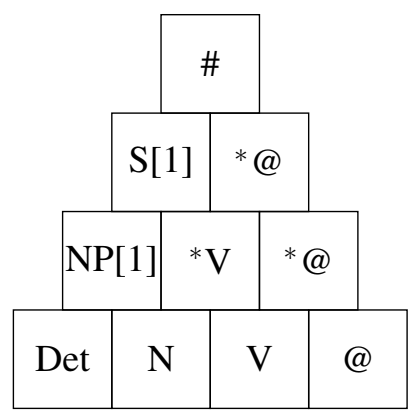

(b) Example 1: 'the dog eats'

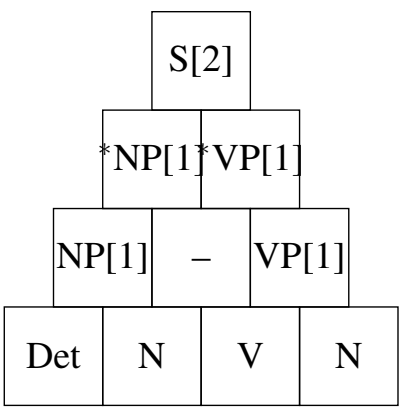

(c) Example 2: 'the dog eats chicken'

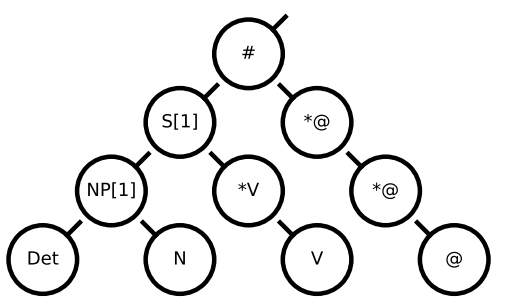

(d) Example 1

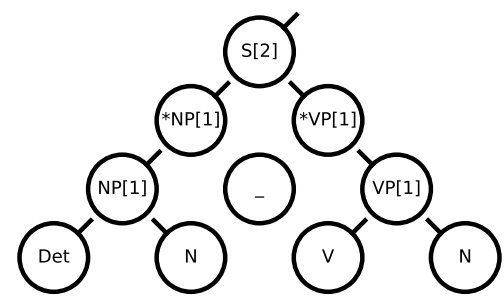

(e) Example 2

Figure 2: Brick roles, with parent-child dependencies shown by lines connecting symbols. (a) Stacks of brick roles. The $p$-th role (from left to right) of the $l$-th layer (from bottom to top) is labeled $(l, p)$. (b, d) Representation of S[1](NP[1](Det, N), V). (c, e) Representation of S[2](NP[1](Det, N), VP[1](V, N)). Symbol *X denotes a copy of X. Symbols \#, @, and _ are introduced to fill unused roles. See text for more details.

corresponds to both the left child position of $(l+1, p)$ and the right child position of $(l+1, p-1)$. Thus role $(l, p)(l \geq 2)$ has $(l-1, p)$ as its left child and $(l-1, p+1)$ as its right child. In this way, brick roles can represent parent-child relationships in a tree structure. Importantly, fillers bound to a brick role can interact with fillers bound to another brick role only when the two brick roles form the direct parent-child relationship.

Note that brick roles are constructed in a bottom-up fashion from terminal brick roles $(1, p)$. The underlying motivation is that for any input word in a sentence, the position information is available to the parser. For example, if an input sentence is 'Det N V', the parser knows that fillers Det, N, and V must be bound to terminal brick roles $(1,1),(1,2)$, and $(1,3)$, respectively. Given a maximum sentence length $N_{\max }$, the number of terminal roles is set to $N_{\max }$, which results in a set of $N_{\max }\left(N_{\max }+1\right) / 2$ brick roles.

The restriction of interactions to direct parent-child relationships is, in some cases, insufficient to properly represent trees with embedding. For example, Figures $2 b$ and $2 d$ present the representation of $\mathrm{S}[1](\mathrm{NP}[1]($ Det, $\mathrm{N}), \mathrm{V})$. In this example, $\mathrm{V}$ is the right child of $\mathrm{S}[1]$ but the roles that they occupy, $(1,3)$ and $(3,1)$, do not have direct interactions, preventing a proper representation of this parent-child dependency. To address this issue, we introduce copy symbols (e.g., $\left.{ }^{*} \mathrm{~V}\right)$ through which the parent-child pair can interact; $\mathrm{S}[1] /(3,1)$ interacts with $* \mathrm{~V} /(2,2)$, which in turn interacts with $\mathrm{V} /(1,3)$.

In addition, we introduce symbols @ and \# to deal with sentences of variable lengths, augmenting the 
parse tree as follows: \#(S[1](NP[1](Det, N), V), @ ). ${ }^{9}$ The padding symbol @ fills empty terminal roles after the sentence-final word. \# has either a root symbol (such as S[1]) or itself (\#) as its left child, and @ as its right child (via*@'s as needed). To illustrate, if the maximum sentence length is 5, the example structure will be augmented as follows: \#(\#(S[1](NP[1](Det, N), V), @), @).

In addition to augmenting short sentences, we must allow for some brick roles to be empty within a parse tree. For example, Figures $2 \mathrm{c}$ and 2e present the representation of S[2](NP[1](Det, N), VP[1](V, N)) where ',' denotes the null filler symbol that binds to the to-be-empty role which does not have a parent nor a child. Note also how symbols in non-adjacent brick roles, like NP[1] and VP[1], via their copies, can combine with $\mathrm{S}[2]$ to form a constituent.

Finally, recall that brick role $(l, p)$ corresponds to both the left child of $(l+1, p)$ and the right child of $(l+1, p-1)$. To make parsing more robust to local ambiguity, we introduce branching-specific filler symbols to distinguish between two instances of the same symbol that play different roles in a local parse tree. More specifically, X:0 and X:1 denote $\mathrm{X}$ as a left child and $\mathrm{X}$ as a right child, respectively. ${ }^{10}$ By using branching-specific fillers, the model is forced to make a micro-parsing decision in each brick role (see below for more details). This allows the model to more easily parse strings with local ambiguity. For ease of discussion, we will omit these branching-specific notations until the simulation results are presented.

\subsubsection{Vector representation of tree structures}

Given the tree template consisting of a finite number of brick roles, any parse tree instance can be viewed as a set of filler/role bindings (Newell, 1980; Smolensky, 1990). For example, the tree in Figure 2b is (assuming $N_{\max }=4$, as in Figure 2): S[1](NP[1](Det, N), V) $\equiv\{\operatorname{Det} /(1,1), \mathrm{N} /(1,2), \mathrm{V} /(1,3), @ /(1,4), \mathrm{NP}[1] /(2,1)$, $\left.{ }^{*} \mathrm{~V} /(2,2),{ }^{*} @ /(2,3), \mathrm{S}[1] /(3,1),{ }^{*} @ /(3,2), \# /(4,1)\right\}$. As previewed above, we follow Smolensky (1990) and map a set of discrete symbolic structures to a continuous vector space as follows. Let $\mathscr{F}=\left\{F_{1}, F_{2}, \cdots, F_{N_{F}}\right\}$ and $\mathscr{R}=\left\{R_{1}, \cdots, R_{N_{R}}\right\}$ be the set of filler symbols and the set of brick roles, respectively, where $N_{F}$ and $N_{R}$ are the numbers of fillers and roles. Let $\mathbf{f}_{i}$ and $\mathbf{r}_{j}$ be the vector representations of filler $F_{i}$ and role $R_{j}$. The tensor product of the filler and the role vectors is taken as the representation of the binding: $F_{i} / R_{j} \equiv \mathbf{f}_{i} \otimes \mathbf{r}_{j}$. The vector representation of the whole structure is simply the superposition (or sum) of the binding vectors. For any parse tree, we have $\mathrm{S} \equiv\left\{F_{f_{\mathrm{S}}\left(R_{1}\right)} / R_{1}, \cdots, F_{f_{\mathrm{S}}\left(R_{N}\right)} / R_{N_{R}}\right\}$, where $f_{\mathrm{S}}\left(R_{k}\right)$ returns the index of the filler bound to role $R_{k}$ in $\mathrm{S}$; then $\mathrm{S} \equiv \sum_{k=1}^{N_{R}} \mathbf{f}_{\mathrm{f}_{\mathrm{S}}\left(R_{k}\right)} \otimes \mathbf{r}_{k}$.

Filler vectors and role vectors are assumed to satisfy the following constraints: (a) every vector has unit length and (b) filler vectors are linearly independent of each other, as are role vectors-thus, binding vectors are linearly independent as well. Linear independence is necessary to prevent a superposition catastrophe; for each role $R_{k}, F_{f_{\mathrm{S}}\left(R_{k}\right)}$ is precisely recoverable from the vector representation of a whole structure $\mathrm{S}$ when, and only when, role vectors are linearly independent (Smolensky, 1990). In addition, pairwise similarity

\footnotetext{
${ }^{9}$ Unlike Stevenson (1994), Vosse and Kempen (2000), Tabor and Hutchins (2004) where the representation space is expanded dynamically with every new word input, our model has a static representation space.

${ }^{10}$ Each branching-specific filler X:r can be viewed as a binding of filler $\mathrm{X}$ with role $\mathrm{r}$ where $\mathrm{r}$ denotes the child position with respect to its direct parent. For example, $\mathrm{X}: 0$ denotes $\mathrm{X}$ as the first child of its parent, and its vector representation is defined as $\mathbf{f}_{\mathrm{X}} \otimes \mathbf{r}_{0} ; \mathrm{X}: 1$ is defined as $\mathbf{f}_{\mathrm{X}} \otimes \mathbf{r}_{1}$. In the present study, we treat X:0 and X:1 as two different filler symbols by setting their pairwise representational similarity to 0 . Root symbols are bound to role 0 for convenience. For simplicity our simulations exclude unnecessary branching-specific fillers (e.g., Y:0 if Y can occur only as a right child of its parent).
} 
among fillers is modeled abstractly by constraining the dot product of two filler vectors to a specified value in the interval $[0,1)$; likewise for roles. ${ }^{11}$ In practice, the GSC model may have trouble when representation similarity is too high. We reserve the question of how processing dynamics is influenced by representational similarity for future studies.

Given $N_{F}$ fillers and $N_{R}$ roles, we consider a dynamical system consisting of $N=N_{F} N_{R}$ state variables (or processing units): the minimal dimension of a tensor product representation with linearly independent fillers and roles. The elements of the state vector will be indexed by a pair consisting of a filler index and a role index. Let $\mathbf{a} \in \mathbb{R}^{N_{F} N_{R}}$ be an activation state of the system in a coordinate-free form. We use two coordinate systems: neural and conceptual coordinates (Smolensky, 1986). The neural coordinates of an activation state are the activation values of the individual network units; these can be taken as the components of the activation vector relative to the standard basis in the tensor-product space, consisting of vectors $\mathbf{e}^{\phi \rho}=\mathbf{e}_{F}^{\phi} \otimes \mathbf{e}_{R}^{\rho}$ with components 1 in position $\phi \rho$ otherwise 0 (for $\phi=1, \ldots, N_{F} ; \rho=1, \ldots, N_{R}$ ). ${ }^{12}$ In neural coordinates, the state vector is expressed as: $\mathbf{a}_{\mathbf{n}} \equiv \mathbf{n} \equiv\left(\cdots, n_{\phi \rho}, \cdots\right)=n_{\phi \rho} \mathbf{e}^{\phi \rho}$ where here and henceforth we use the Einstein summation convention, implicitly summing over the repeated indices $\phi, \rho$. In conceptual coordinates, the activation state is expressed as: $\mathbf{a}_{\mathbf{c}} \equiv \mathbf{c} \equiv\left(\cdots, c_{f r}, \cdots\right)=c_{f r} \hat{\mathbf{e}}^{f r}$ where $\hat{\mathbf{e}}^{f r}=\hat{\mathbf{e}}_{F}^{f} \otimes \hat{\mathbf{e}}_{R}^{r}$ are the conceptual basis vectors, with $\hat{\mathbf{e}}_{F}^{f}$ the vector representing filler $f$ and $\hat{\mathbf{e}}_{R}^{r}$ the vector representing role $r$ (for $f=1, \ldots, N_{F} ; r=$ $\left.1, \ldots, N_{R}\right) . \mathbf{n}$ and $\mathbf{c}$ can be viewed as being built respectively from the distributed and the local representations of bindings $\left\{F_{f} / R_{r}\right\}$. Let $\mathbf{N}$ be the $N \times N$ matrix with element in row $\phi \rho$ and column $f r$ equal to the $\phi \rho^{\text {th }}$ neural coordinate of the vector $\hat{\mathbf{e}}^{f r}$ encoding $F_{f} / R_{r}$ : the product of the $\phi^{\text {th }}$ neural coordinate of $\hat{\mathbf{e}}_{F}^{f}$ and the $\rho^{\text {th }}$ neural coordinate of $\hat{\mathbf{e}}_{R}^{r}$. Then $\mathbf{N}$ can be used to change basis in the following way ${ }^{13}: \mathbf{n}=\mathbf{N c}$ and $\mathbf{c}=\mathbf{N}^{-1} \mathbf{n}$. $\mathbf{D}=\mathbf{N}^{\top} \mathbf{N}$ is the matrix of pairwise binding similarities.

An activation state $\mathbf{a}$ is referred to as a grid point if its conceptual coordinates satisfy the following condition: in c, for every $r$ there exists $f_{r}$ such that $c_{f r}=1$ if $f=f_{r}$, and $c_{f r}=0$ otherwise. This states the condition that each role binds exactly one filler ${ }^{14}$. Grid points are taken as the vector representation of (either grammatical or ungrammatical) combinatorial discrete symbolic structures.

\subsection{Constraints and Harmony functions}

The GSC model is designed to optimize grammatical and non-grammatical constraints. Non-grammatical constraints collectively pursue some balance between the unbiased blend state (where every filler is equally partially active) and each of the grid points. Non-grammatical constraints are applied to each role independently, treating all grid points as well-formed symbolic states. Grammatical constraints introduce biases

\footnotetext{
${ }^{11}$ One way of representing symbol similarity is to use feature-based representations. For example, the representation of a lexical category $\mathrm{N}$ can be a vector where each component (corresponding to a single processing unit) encodes the value of a particular feature (e.g., '+plural') (e.g., Smith et al., 2018). We note that: (1) the feature-based representation of symbols is a special case of tensor product variable binding where features can be thought of as roles and '+', '-' as fillers; and (2) our dot-product based approach makes it possible to study the effect of representation similarity abstractly without choosing a particular set of features. In such a feature-based approach, choosing orthonormal filler and role vectors entails that the dot-product similarity of two symbols equals the number of feature values they share.

${ }^{12}$ Here and below, each element of an activation vector in $\mathbb{R}^{N}$ that is described in neural coordinates is indexed by a pair $(\phi, \rho)$, which we abbreviate $\phi \rho$. Likewise, $f r$ abbreviates a pair that indexes an element of a vector described in conceptual coordinates.

${ }^{13} \mathbf{n}=\mathbf{N c}$ means $\mathbf{n}_{\phi \rho}=\mathbf{N}_{\phi \rho}^{f r} \mathbf{c}_{f r}$, with summation over $f, r$.

${ }^{14}$ Unused or "empty” roles are filled by '_, ' '@', '\#’ or their copy symbols.
} 
favoring grammatical grid points (i.e., grid points optimally satisfying the grammatical constraints) over ungrammatical grid points.

For each of these constraints, a Harmony function is defined to map activation states to real Harmony values, the graded measures of goodness with respect to the constraint. Each Harmony function is continuous and smooth, and has maximum values at the activation states that maximally satisfy the relevant constraints. Then, the Total Harmony function is defined as the sum of the component Harmony functions. For geometric intuition, we will use minimalistic examples to visualize the shape of the Harmony functions (called Harmony surfaces) on a subset of the representation space.

\subsubsection{Non-grammatical constraints}

We consider in turn the three non-grammatical constraints in the model. First, the Resource Constraint restricts the amount of computational resource available for each role to a finite value. More specifically, the sum-of-squares of filler activations in each role is restricted to 1 via the following Resource Harmony function:

$$
H_{R}\left(\mathbf{a}_{\mathbf{c}} ; m\right)=-m \sum_{r}\left(1-\sum_{f} a_{f r}^{2}\right)^{2}
$$

where $f$ and $r$ are filler and role indices, $a_{f r}$ is the activation value of binding $\mathrm{F} / \mathrm{R}$, and a free parameter $m$ is a scaling factor.

To illustrate this, assume that terminal role fillers are individual lexical items ('the'), rather than grammatical categories ('Det'). We'll examine a grammar that allows for three initial noun phrases, denoted with brackets (discussed in more detail below; NP[1]: 'the dog'; NP[2]: 'dog with spots'; NP[3]: 'big dog'). The first panel of Figure 3 presents the Harmony surface on a subspace spanning two bindings in the first position of the sentence: the/(1,1) and $\operatorname{dog} /(1,1)$ (assume the activation values of all the other fillers in role $(1,1)$ are 0$)$. The set of maxima in this subspace is presented as an arc in the first panel in the figure. We will refer to this subspace as a resource manifold in role $r, M_{r}$. More generally, this manifold will be the surface of a unit hypersphere (see the first panel in Figure 4). The set of maxima in the full activation state space is the product space of these manifolds: $M \equiv \Pi_{r} M_{r}$.

Second, the Baseline Constraint favors an unbiased, balanced blend state of fillers in each role over biased blend states via the Baseline Harmony function:

$$
H_{B}\left(\mathbf{a}_{\mathbf{c}} ; \mathbf{z}_{\mathbf{c}}, \beta\right)=-\frac{\beta}{2}\left(\mathbf{a}_{\mathbf{c}}-\mathbf{z}_{\mathbf{c}}\right)^{\top}\left(\mathbf{a}_{\mathbf{c}}-\mathbf{z}_{\mathbf{c}}\right)
$$

where $\mathbf{z}_{\mathbf{c}}$ is the baseline state (in conceptual coordinates) and a free parameter $\beta$ is a scaling factor. To respect the Resource Constraint, $\mathbf{z}_{\mathbf{c}}$ is set to $\left(1 / \sqrt{N_{F}}\right) \cdot \mathbf{1}$ where $N_{F}$ is the number of filler symbols. The more distant an activation state is from the baseline state, the worse it is (see the second panels of Figure 3 and 4). (Note that because the baseline state, the maximum of the Baseline Harmony surface, lies on the resource manifold, the maximum of the sum of Resource and Baseline Harmony functions is the baseline state.)

In contrast to the Baseline Constraint, the Discreteness Constraint favors discrete activation states (all 

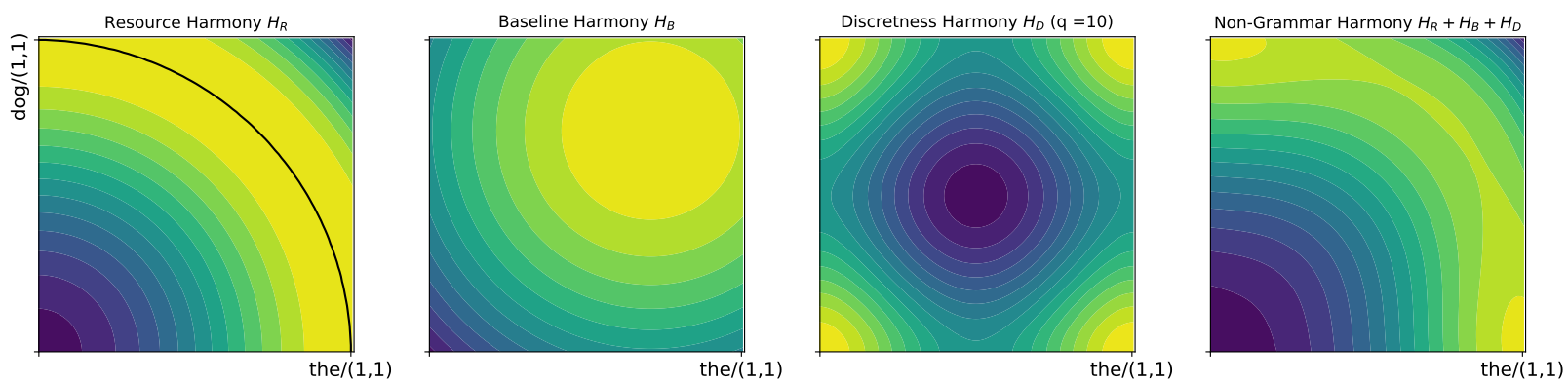

Figure 3: Schematic diagrams of Non-Grammar Harmony surfaces on a 2D subspace spanning two binding vectors (the/(1,1) and $\operatorname{dog} /(1,1))$. The blue-dark to yellow-light color gradient represents the low-to-high continuum of Harmony values (the maximum value for each constraint is 0 ). $q$ indicates the strength of the role-specific contribution of Discreteness Harmony. Let a set of states where Resource Harmony is 0 be a role-specific resource manifold $M_{r}$; the black line in the first panel shows the manifold. When the weight of Resource Harmony is large enough, the Non-Grammar Harmony values of states on the manifold are larger than those of the states away from the manifold.
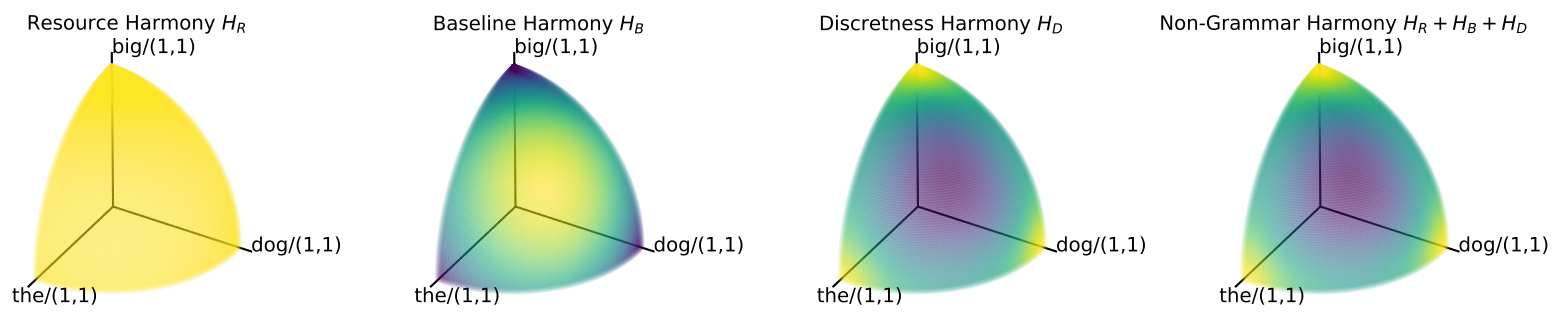

Figure 4: A three-dimensional analogue of Figure 3. The spherical surface in each panel represents the resource manifold connecting three purely discrete states corresponding to the/(1,1), $\operatorname{dog} /(1,1)$, and $\operatorname{big} /(1,1)$. 
fillers either fully active or fully inactive) over states with partial activation of fillers. The Discreteness Harmony function is as follows:

$$
H_{D}\left(\mathbf{a}_{\mathbf{c}} ; \mathbf{q}\right)=-\sum_{f, r} q_{r} a_{f r}^{2}\left(1-a_{f r}\right)^{2}
$$

where each component of $\mathbf{q}=\left(q_{1}, \cdots, q_{r}, \cdots, q_{N_{R}}\right)^{\top}$ specifies a role-specific scaling factor (called commitment strength). The values of each $q_{r}$ change dynamically as a sentence is processed (see Section 3.5.2). Discreteness Harmony surfaces are presented in the third panels of Figure 3 and 4. As shown in Figure 3, the Discreteness Constraint alone does not favor grid points; it prefers discrete activations, but assigns no penalty to activating multiple fillers in a given role (the upper-right corner of the plot). In terms of Discreteness Harmony, two states, $1.0 \cdot$ the $/(1,1)+0.0 \cdot \operatorname{dog} /(1,1)$ and $1.0 \cdot$ the $/(1,1)+1.0 \cdot \operatorname{dog} /(1,1)$, are equally good. The Resource Constraint is critical; it assigns a penalty to co-activation. As shown in the final panels of Figures 3 and 4, the combination of both constraints favors grid points.

As shown in the second and third panels of Figure 4, the Baseline and Discreteness Constraints clearly conflict, with the former preferring an unbiased blend vs. the discrete states preferred by the latter. The relative strength of each of these constraints changes over the course of computation. Initially, commitment strength is low; the contribution of the Discreteness Constraint is set to 0 , favoring an unbiased blend state. As a role-specific commitment strength increases, the maxima of the sum of three Non-Grammar Harmony functions move toward grid points. This is illustrated in the last panels of Figures 3 and 4 . To visualize the dynamics of this change, the panels of Figure 5 illustrates Non-Grammar Harmony at increasing levels of commitment strength.
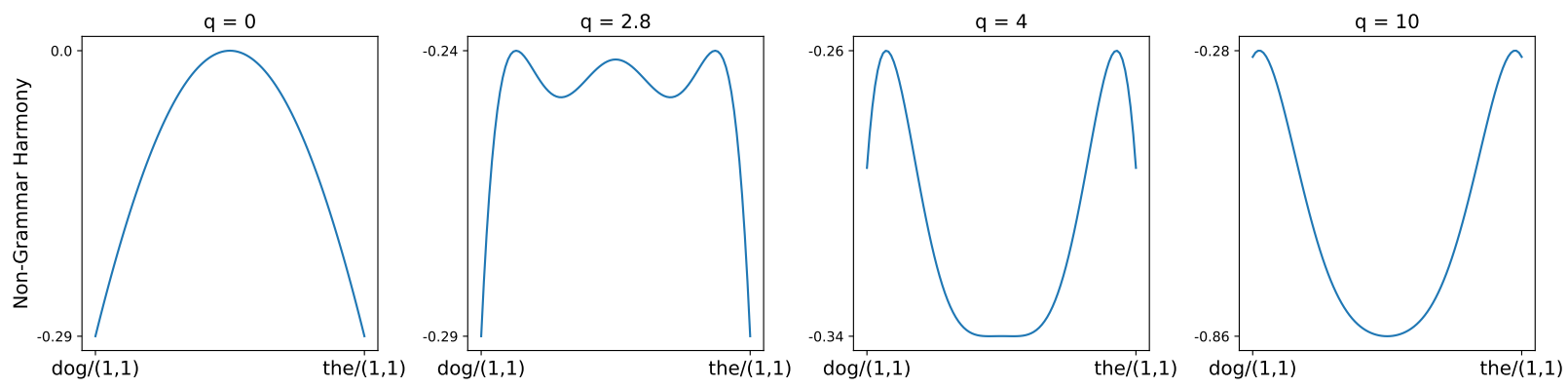

Figure 5: Change to Non-Grammar Harmony surface on the resource manifold, where the Resource Constraint is satisfied (see the arc in the first panel of Figure 3), as a function of commitment level.

All these non-grammatical constraints are applied to different roles independently, allowing all combinatorial combinations of fillers across roles. It is the Grammatical Constraints that introduce the biases toward grammatical combinations of filler/role bindings.

\subsubsection{Grammatical constraints and Harmonic Grammar}

Harmonic Grammar (Hale and Smolensky, 2006; Legendre et al., 1990) is a connectionist-friendly grammar formalism, specifying a symbolic grammar as a set of soft, local constraints and assigning a real number 
(Grammar Harmony) to each constraint when it is satisfied. The well-formedness of any combinatorial structure is quantified as the sum of Harmony values assigned by the constraints that are relevant to that structure. The Harmony values assigned by local constraints can be implemented as the values of weight and bias parameters of a neural network model that represents symbolic structures in its activation states via tensor product variable binding. In this way, Harmonic Grammar provides a way of evaluating the wellformedness of the output of linguistic computation at both symbolic and subsymbolic levels, which is in line with the Integrated Symbolic/Connectionist Cognitive Architecture (Smolensky and Legendre, 2006a,b).

As an example, consider the rule NP $\rightarrow$ Det $\mathrm{N}$, which characterizes a local parse tree NP(Det, N) as grammatical. Harmonic Grammar realizes this rule as a combination of Harmony penalties and rewards (Smolensky, 1993). The occurrence of each symbol in a structure is penalized; a negative value $b$ is assigned to each symbol (e.g., NP, Det, and N). In a grammatical structure, these penalties are compensated for by positive values $w$ assigned to each grammatical parent-child pair (e.g., NP-Det pair, NP-N pair). Then, the Grammar Harmony of a parse tree is simply the sum of all of these Harmony values. Smolensky (1993) chose $w$ and $b$ values so that they would exactly cancel out; every grammatical structure has a Harmony value of 0 and different ungrammatical structures have different negative Harmony values depending on how many local constraints are violated. In this study, we will take a weaker position that every grammatical structure must have a greater Harmony value than every ungrammatical structure. We will use a heuristic training algorithm (see Section 3.7) to find good values of the $w$ and $b$ parameters.

The brick role system provides a clear framework for stating context-free Harmonic Grammar rules as follows. The well-formedness (or Grammar Harmony) between NP as a parent and $\mathrm{N}$ as its left child is $w_{1}$ : $H_{G}(\mathrm{NP} /(l, p)$, Det $/(l-1, p))=w_{1}$. The parent-left child relation is captured by the relation between two brick roles $(l, p)$ and $(l-1, p)$. The $w_{1}$ value can be same for all possible values of $l$ and $p$, making the relation between two filler symbols context-free. In other words, the Harmony of the symbol pair does not depend on where in the tree template the pair occurs.

Because Harmonic Grammar only considers the well-formedness of pairs of symbols, it requires a different representation of grammar rules that allow for variable expansions of a non-terminal. Consider the case where NP can expand to different pairs of symbols: NP $\rightarrow$ Det N ('the dog') | N P[repositional]P[hrase] ('dog with-spots'). For simplicity, we assume here the same value $w$ is assigned to all four parent-child pairs, $b_{\text {terminal }}$ is assigned to all children, and $b_{\text {parent }}$ is assigned to NP. Because Harmony is sensitive only to pairwise relationships between symbols, this set of rules will assign the same Harmony value to both grammatical $(\mathrm{NP}($ Det, $\mathrm{N})$ and $\mathrm{NP}(\mathrm{N}, \mathrm{PP}))$ and ungrammatical parse trees $(\mathrm{NP}($ Det, $\mathrm{PP})$ and $\mathrm{NP}(\mathrm{N}$, $\mathrm{N})$ ). For example, $\mathrm{NP}($ Det, $\mathrm{N})$ has a harmony of $H_{G}(\mathrm{NP} /(2,1)$, Det $/(1,1))+H_{G}(\mathrm{NP} /(2,1), \mathrm{N} /(1,2))+$ $H_{G}(\mathrm{NP})+H_{G}(\mathrm{Det})+H_{G}(\mathrm{~N})=2 w+b_{\text {parent }}+2 b_{\text {terminal }}$. Ungrammatical NP(Det, PP) has the same harmony: $H_{G}(\mathrm{NP} /(2,1), \operatorname{Det} /(1,1))+H_{G}(\mathrm{NP} /(2,1), \mathrm{PP} /(1,2))+H_{G}(\mathrm{NP})+H_{G}(\operatorname{Det})+H_{G}(\mathrm{~N})=2 w+b_{\text {parent }}+2 b_{\text {terminal }}$. To avoid this problem, Smolensky (1993) (see also Hale and Smolensky (2006)) proposed the Harmonic Normal Form transformation of (the Chomsky Normal Form of) a context-free grammar. It replaces binarybranching rules $\{\mathrm{NP} \rightarrow$ Det N $\mid \mathrm{N}$ PP $\}$ with $\{\mathrm{NP} \rightarrow \mathrm{NP}[1] \mid \mathrm{NP}[2], \mathrm{NP}[1] \rightarrow$ Det N, NP[2] $\rightarrow \mathrm{N} \mathrm{PP}\}$. The bracketed symbols NP[k] can be viewed as alternate forms of NP, each expanding to unique pairs of children. 
There are two issues with using this Grammatical Constraint schema for a scalable GSC parser. The introduction of the additional unary-branching rules (e.g., NP $\rightarrow \mathrm{NP}[1]$ ) increases the distance (the number of edges in the path) from a terminal symbol to a root symbol in a parse tree. This increased distance makes parsing more difficult, as key parts of the grammatical tree are less able to interact with one another. Critically, unary branching requires a significant change in the brick role representation of the tree template. For these reasons, we do not use the unary branching rules and replace a non-terminal symbol occurring on the right-hand side of each rewrite rule with bracketed symbols. For example, consider a set of rewrite rules $\{\mathrm{S} \rightarrow \mathrm{NP}$ VP, NP $\rightarrow$ Det N|N Prep, VP $\rightarrow$ V N $\}$. After the Harmonic Normal Form transformation, we get $\{\mathrm{S}[1] \rightarrow \mathrm{NP}[1] \mathrm{VP}[1] \mid \mathrm{NP}[2] \mathrm{VP}[1], \mathrm{NP}[1] \rightarrow$ Det N, NP[2] $\rightarrow$ N Prep, VP[1] $\rightarrow$ V N $\}$. Note that now $\mathrm{S}[1]$ has multiple expansions but it is not a problem any longer because every combination of grammatical left-children and right-children is grammatical.

Figure 6 illustrates how HG rules are implemented as the weights of connections in a localist neural network where the activation values of the nodes can be viewed as the conceptual coordinates of the activation vector. (n.b. This approach generalizes fully to distributed representations.) For simplicity, we assume that the binding vectors are orthogonal. The Grammar Harmony of a single binding (e.g., Det/(1,1)) is defined as its activation level squared times its unary Harmony $b: b \cdot a(\operatorname{Det} /(1,1))^{2}$. The Grammar Harmony of a binding pair (e.g., Det/(1,1) and NP[1]/(2,1)) is defined as the product the their activation levels multiplied by the binary Harmony value $w: w \cdot a(\operatorname{Det} /(1,1)) \cdot a(\mathrm{NP}[1] /(2,1))$. When an external input is given to a binding, the Grammar Harmony of the binding with respect to the external input is defined as the product of the binding's activation level and the external input strength $x: x(\operatorname{Det} /(1,1)) \cdot a(\operatorname{Det} /(1,1))$. Note that these Harmony Grammar functions naturally extend HG rules (which apply to discrete symbolic states [or grid points]) to continuous activation states. The Grammar Harmony of the global activation state is simply the sum of the Grammar Harmony values of bindings and binding pairs.

For example, using the numerical values shown in Figure 6, the Grammar Harmony of the activation state $(0.5,0.4)^{\top}$ is as follows: $w \cdot a(\mathrm{NP}[1] /(2,1)) \cdot a(\operatorname{Det} /(1,1))+b \cdot a(\operatorname{Det} /(1,1)) \cdot a(\operatorname{Det} /(1,1))+b \cdot a(\mathrm{NP}[1] /(2,1))$. $a(\mathrm{NP}[1] /(2,1))+x(\operatorname{Det} /(1,1)) \cdot a(\operatorname{Det} /(1,1))=2 \cdot 0.5 \cdot 0.4-1 \cdot 0.5 \cdot 0.5-1 \cdot 0.4 \cdot 0.4+2 \cdot 0.5=1.09$. More succinctly, the Grammar Harmony is $(1 / 2) \mathbf{a}_{\mathbf{c}}^{\top} \mathbf{W}_{\mathbf{c}} \mathbf{a}_{\mathbf{c}}+\mathbf{x}_{\mathbf{c}}^{\top} \mathbf{a}_{\mathbf{c}}$ where $\mathbf{a}_{\mathbf{c}}=(0.5,0.4)^{\top}$ is the activation state, $\mathbf{x}=(2,0)^{\top}$ is the external input vector, and $\mathbf{W}_{\mathbf{c}}=[[2 b, w],[w, 2 b]]=[[2,-2],[-2,2]]$ is the weight matrix in conceptual coordinates.

More generally, let $\mathbf{W}_{\mathbf{c}}$ be the weight matrix where the $\left(i j, i^{\prime} j^{\prime}\right)$-th component specifies either the Grammar Harmony between bindings $F_{i} / R_{j}$ and $F_{i^{\prime}} / R_{j^{\prime}}$, if $i j \neq i^{\prime} j^{\prime}$, or two times the Grammar Harmony of the individual binding $F_{i} / R_{j}$, if $i j=i^{\prime} j^{\prime}$. The Grammar Harmony of an activation state, described in neural coordinates as $\mathbf{n}$, is defined as follows:

$$
H_{G}(\mathbf{n})=\frac{1}{2} \mathbf{n}^{\top} \mathbf{W}_{\mathbf{n}} \mathbf{n}+\mathbf{x}_{\mathbf{n}}^{\top} \mathbf{n}=\frac{1}{2} \mathbf{c}^{\top}\left(\mathbf{N}^{\top} \mathbf{W}_{\mathbf{n}} \mathbf{N}\right) \mathbf{c}+\mathbf{x}_{\mathbf{c}}^{\top}\left(\mathbf{N}^{\top} \mathbf{N}\right) \mathbf{c}
$$

where $\mathbf{n}, \mathbf{x}_{\mathbf{n}}$, and $\mathbf{W}_{\mathbf{n}}$ are the activation state, the external input vector, and the weight matrix in neural coordinates; as discussed in Section 3.1.2, $\mathbf{N}$ is the change of basis matrix such that $\mathbf{n}=\mathbf{N c}$, and correspondingly $\mathbf{W}_{\mathbf{n}}=\left(\mathbf{N}^{-1}\right)^{\top} \mathbf{W}_{\mathbf{c}} \mathbf{N}^{-1}$. The above Harmony function provides a way of evaluating the well-formedness of any activation state. 


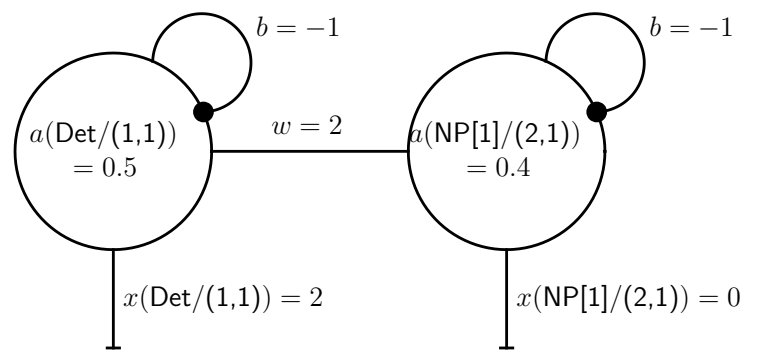

Figure 6: An example representation of grammatical constraints in a graph. $a(\mathrm{f} / \mathrm{r})$ is the activation level of a binding $\mathrm{f} / \mathrm{r}, w$ is the Grammar Harmony of the pair of bindings, $b$ is the Grammar Harmony of each individual binding, and $x(\mathrm{f} / \mathrm{r})$ is the external input to binding $\mathrm{f} / \mathrm{r}$.

To gain geometrical insights into the Grammar Harmony surface, we need to consider a subspace spanning two bindings employing two different roles (e.g., Det/(1,1) and NP/(2,1)). For this purpose, we consider a one-dimensional subspace for each role space. This one-dimensional space is a spherical linear interpolation of the baseline state (where every filler is equally partially active) and a discrete state (where one filler is fully active and all the other fillers are inactive) in each role. Recall from above that the Resource Constraint biases activation states to a manifold where the Resource Constraint assigns high harmony (illustrated in Figure 4). Figure 7 presents one-dimensional subspaces along these resource manifolds from each of two role spaces. One extreme of the subspace is the maximum value of Non-Grammar Harmony when commitment strength is $0: z\left(r_{i}\right)$, the (conceptual) coordinates of the baseline vector in each role space. The other extremes are the maximum value of Non-Grammar Harmony when commitment strength is high: the coordinates of different grid points in each role space. For example, the spherical linear interpolation from $z((1,1))$ to $\operatorname{Det} /(1,1)$ on the role-specific resource manifold $M_{(1,1)}$, denoted as $M_{(1,1)}^{\text {Det }}$, reflects the gradual change from the unbiased blend in the $(1,1)$ role towards the grid point $\operatorname{Det} /(1,1)$ while respecting the resource constraint.

Figure 8 presents the Grammar Harmony surfaces on different subspaces (the product spaces $M_{(1,1)}^{f_{i}} \times$ $\left.M_{(2,1)}^{f_{j}}\right)$ in different columns. ${ }^{15}$ Recall that we are examining a grammar that allows for three initial noun phrases (NP[1]: 'the dog'; NP[2]: 'dog with spots'; NP[3]: 'big dog'). The first and last column show grammatical binding pairs: $(\operatorname{Det} /(1,1), \mathrm{NP}[1] /(2,1))$ and $(\mathrm{N} /(1,1), \mathrm{NP}[2] /(2,1))$, respectively. The middle column shows the ungrammatical binding pair (Det/(1,1), NP[2]/(2,1)). As shown in the top row, in the absence of external input, coactivation of grammatical pairs of bindings (first and third columns) is encouraged: the upper-right corner has high Harmony; co-activation of ungrammatical pairs is however discouraged (middle column): the upper-right corner has low Harmony. As shown in the bottom row, an external input to $\operatorname{Det} /(1,1)$ introduces a bias toward coactivation of a grammatical pair consistent with the input (first column: upper-right corner has high Harmony) over coactivation of alternative grammatical pairs inconsistent with

\footnotetext{
${ }^{15}$ Note that, unlike Figure 3, in Figure 4 the lower-left corner is the baseline state $(z, z)$, not $(0,0)$.
} 

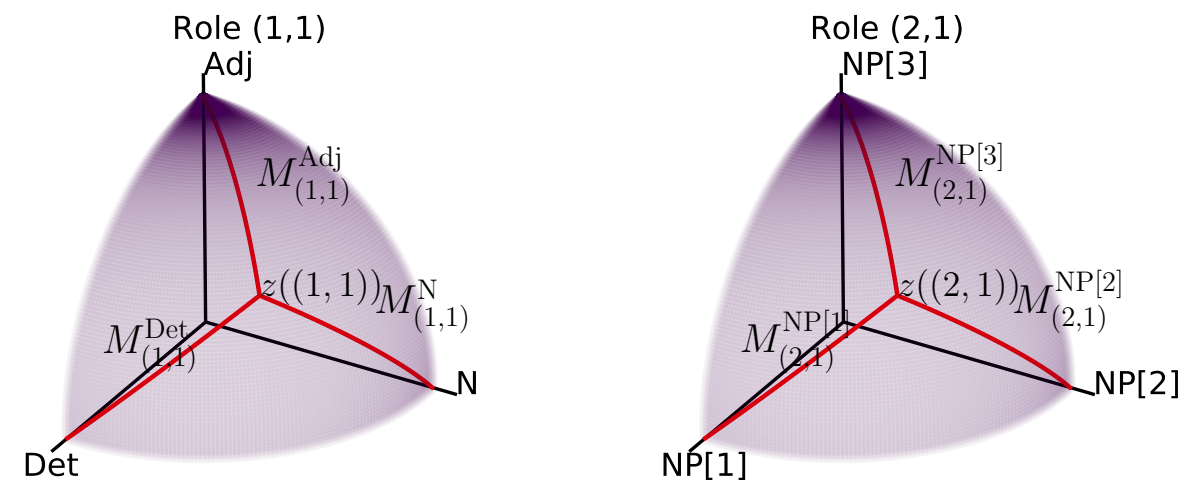

Figure 7: Spherical surfaces in two subspaces each of which spans fillers bound to role $(1,1)$ and $(2,1)$, respectively. The red lines on the spheres are spherical linear interpolations from the baseline activation state to each grid point. In the following, we consider the product space of the red lines in roles $(1,1)$ and $(2,1)$.

the input (last column: upper-right corner has lower Harmony). Finally, note that the grammatical constraints assign high harmony to states with substantial coactivation of grammatical bindings. In the absence of external input, there is high harmony along the entire diagonal of the subspace in both the first and third columns, although the exact shape of the Harmony surface depends on parameters $b$ and $w$. In order to yield discrete outputs, it is necessary for these constraints to combine with Non-Grammatical Constraints that prefer discrete outputs.

\subsubsection{Total Harmony}

Non-Grammatical and Grammatical constraints combine to determine Total Harmony, the graded wellformedness measure of an activation state given all the above constraints:

$$
H(\mathbf{a})=H_{G}(\mathbf{a} ; \mathbf{W}, \mathbf{x})+H_{B}(\mathbf{a} ; \beta, \mathbf{z})+H_{R}(\mathbf{a} ; m)+H_{D}(\mathbf{a} ; \mathbf{q})
$$

where $\beta$ and $m$ are free parameters fixed at constant values. As assumed in the discussion above, $m$ is large enough that the Resource constraint effectively restricts activation to states close to the resource manifold $M=\Pi_{r} M_{r}$. While parsing, the role-specific commitment levels $q_{r}$ then force the model to choose a filler over the others in role $R_{r}$. Each $q_{r}$ is treated as a control parameter that changes the Harmony surface dynamically. We refer to $\mathbf{q}$, the vector of $q_{r}$ 's, as the commitment state.

Continuing the example above, Figure 9 illustrates how the Total Harmony surface on a subspace changes under the external input to binding Det/ $(1,1)$ as a function of the commitment state $\left(q_{(1,1)}, q_{(2,1)}\right)$. The first Total Harmony panel (bottom row, second from left) shows the initial state, where $\mathbf{q}=(0,0)$. Here, the external input biases the Harmony maximum toward the grammatical pair Det/(1,1) and NP[1]/(2,1). Next, as $q_{(1,1)}$ increases from 0 to 5 , the optimal activation state in brick role $(1,1)$ moves toward binding 

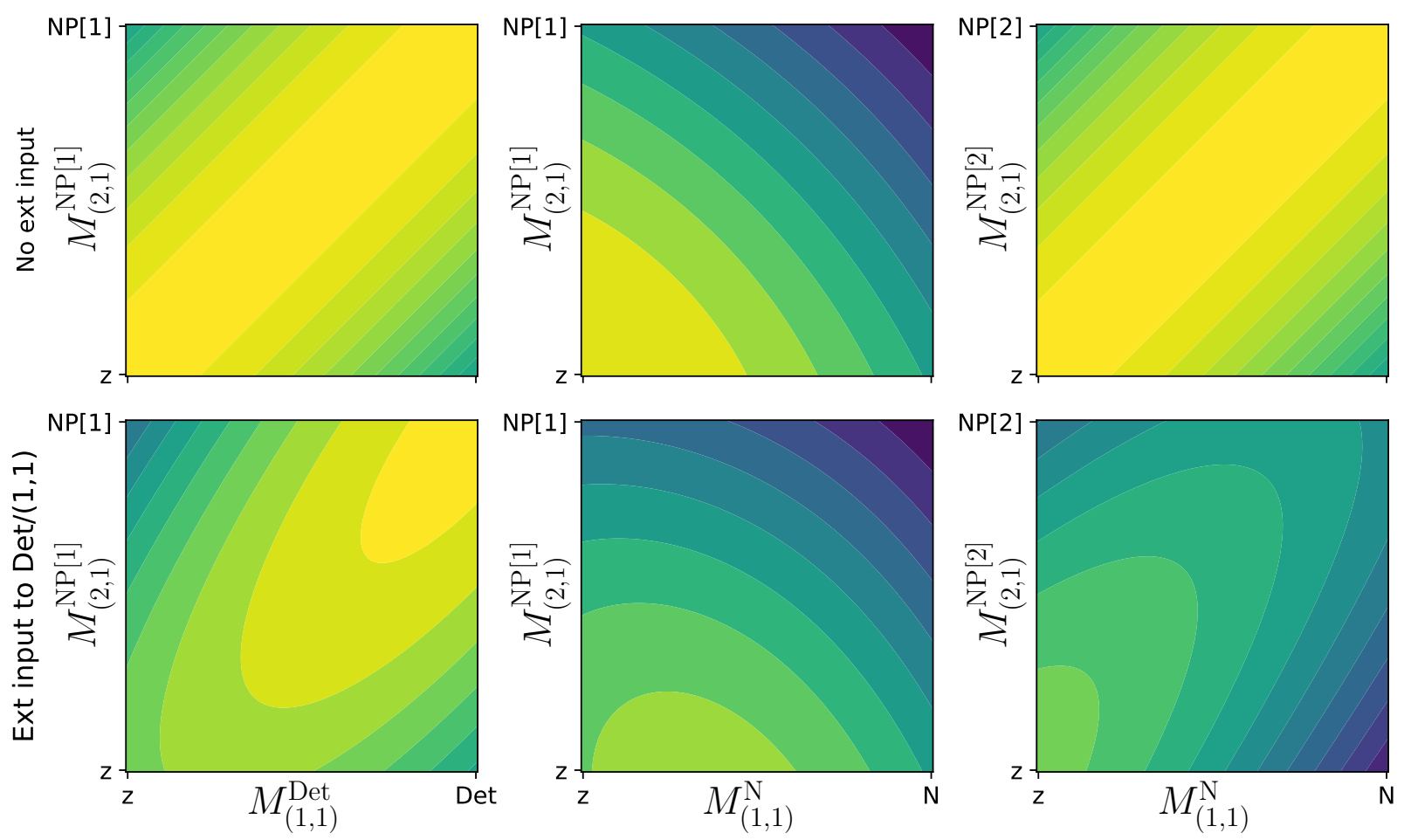

Figure 8: Grammar Harmony surfaces on three subspaces (corresponding to three columns) under different external inputs (top row: no external input, bottom row: external input to binding $\operatorname{Det} /(1,1)$ ).

$\operatorname{Det} /(1,1)$ - the system becomes more committed to the Determiner as the filler in the brick role. At the same time, the optimal activation state in role $(2,1)$ moves toward binding $\mathrm{NP}[1] /(2,1)$ because the grammatical constraint favors coactivation of bindings Det/(1,1) and NP[1]/(2,1). However, because the commitment level in role $(2,1)$ has not increased (and the other Non-Grammatical constraints prefer an unbiased blend state), the displacement relative to the initial state is smaller in role $(2,1)$ than in role $(1,1)$. In the final panel, where $q_{(2,1)}$ changes from 0 to 5 , the optimal activation state on $M_{(2,1)}^{\mathrm{NP}[1]}$ moves much closer to the pure discrete state of binding NP[1]/(2,1). This example illustrates how external input biases the activation state toward context-appropriate grid points and how the model can maintain uncertainty by the control of the commitment state. Section 3.5.2 will present a general dynamic control regime of the commitment state for incremental parsing.

\subsection{Processing dynamics}

In the GSC framework, processing is simply an optimization (or constraint-satisfaction) process. The activation state of the model $\mathbf{a}(t)$, a tensor product representation deploying a set of fillers $\mathscr{F}$ and a set of roles $\mathscr{R}$, changes over time $t$ to maximize the Total Harmony by stochastically following the Harmony gradient (following Movellan, 1998; Movellan and McClelland, 1993):

$$
d \mathbf{a}=\nabla_{\mathbf{a}} H(\mathbf{a}, \mathbf{q}) d t+\sqrt{2 T} d B
$$




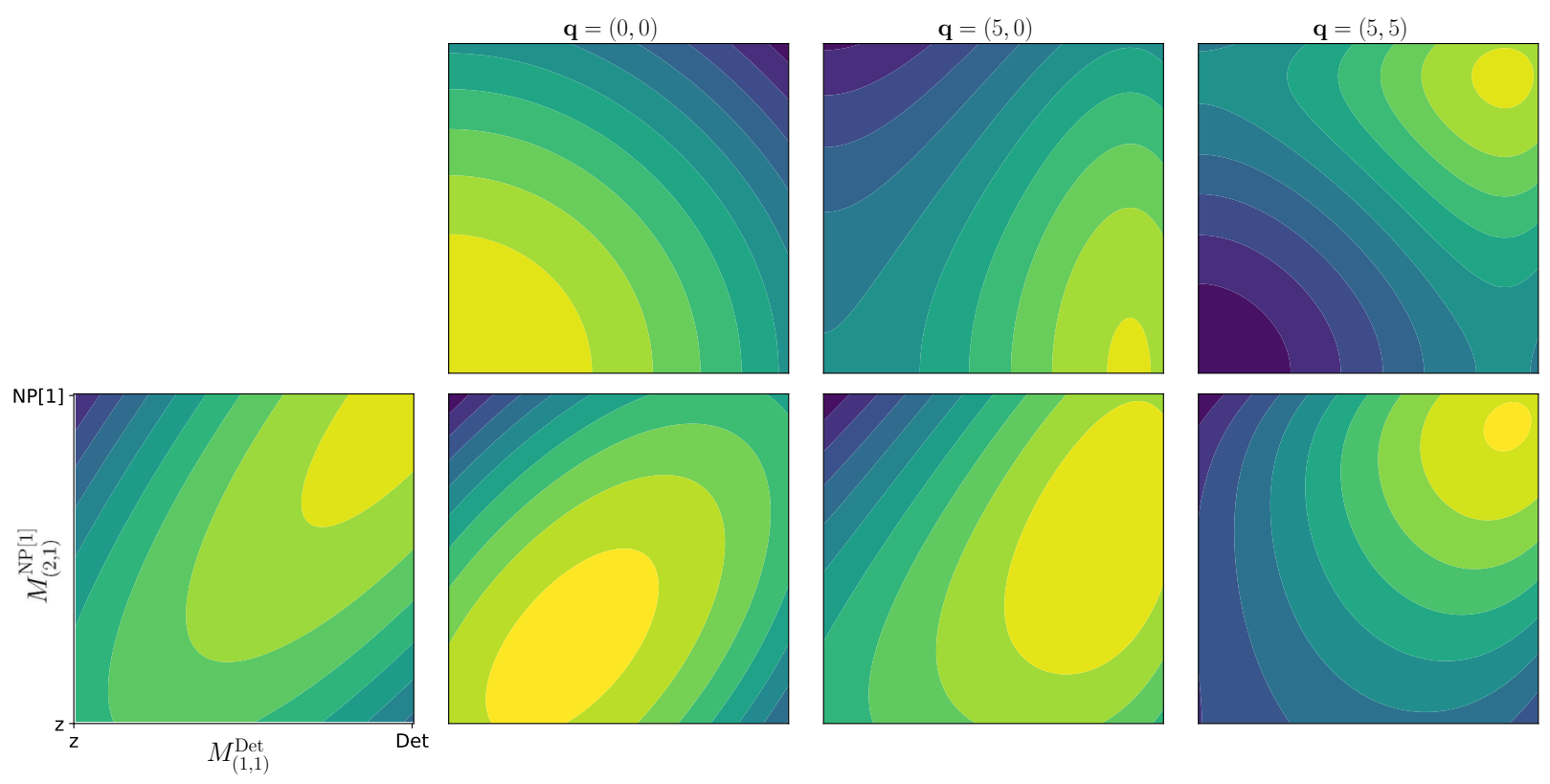

Figure 9: (Top row) Non-Grammar Harmony surfaces on a subspace at different commitment levels. (Bottom row, first panel) Grammar Harmony surface. (Bottom row, remaining panels) Total Harmony surface: sum of Grammar Harmony and the corresponding Non-Grammar Harmony at different commitment states 
where $d B$ is the multi-dimensional Brownian motion and $T$ is a free parameter called computational temperature, which determines the amount of noise in processing dynamics. ${ }^{16}$ The model implementation uses a discrete-time approximation to integrate Eq. 6 in neural coordinates:

$$
\mathbf{a}(t+1)=\mathbf{a}(t)+\nabla_{\mathbf{a}} H(\mathbf{a}(t), \mathbf{q}(t)) \Delta t+\sqrt{2 T \Delta t} \xi
$$

where $\xi \sim N(\mathbf{0}, \mathbf{I})$. In the present study, $T$ is fixed to a relatively small value, following Cho et al. (2017). This is because we are interested in incremental parsing and a large temperature makes the intermediate activation state after processing each word of a sentence unreliable.

The vector $\mathbf{q}(t)$ has one element $q_{r}$ for each role $r \in \mathscr{R}$. We will refer to the method that the model uses to update $\mathbf{q}$ as the commitment policy $\pi: d \mathbf{q}=\pi(\mathbf{a}, \mathbf{q}, \mathbf{x}) d t$. In the present study, we use a relatively simple commitment policy and investigate the model's behavior under the policy. But the GSC model can be augmented with a separate control mechanism implementing policy $\pi$ in a separate neural network. More details are presented in the following sections.

\subsection{Generating complete parse trees: The wrap-up process}

To generate complete parse trees from intermediate model states, we implement a wrap-up process. The model's commitment strength $q_{r}$ is increased for every role $r$ at the rate $d q_{r} / d t=1$ until it reaches the parameter value $q_{\max }$. By applying this process, the model makes a parsing decision given the information available when the wrap-up process begins. The output is a nearly-discrete state close to a grid point representing a parse tree. Typically, but not necessarily, in each role, the most active filler at the start of the wrap-up process will be chosen as the winner.

The wrap-up process is required because a grammatical sentence can be nested in another grammatical sentence. For examples, "The dog eats" and "The dog eats chicken" are both grammatical. Ideally, at the end of incremental parsing of the second word "eats", the model must maintain uncertainty between these sentences (and others). The model can know "eats" is the final word of the first sentence only when it is not followed by another word. When "eats" is in fact not followed by another word, the model initiates the wrap-up process to choose a parse tree corresponding to the first sentence, not the second sentence. As discussed above, in our model the absence of a following word input is modeled by the presence of a special empty-position symbol '@'.

The wrap-up process can also be used to map a GSC state, a pair $(\mathbf{a}, \mathbf{q})$, to a probability distribution over complete parse trees. We estimate the distribution by repeatedly applying the wrap-up process to the same GSC state many times; our estimate is based on the relative frequencies of the resulting parse trees.

As we discuss in more detail below, if the wrap-up process is applied at the start of computation, it can be viewed as a sentence production process. We will use this idea to train a GSC model to approximate a probabilistic context-free grammar for sentence production.

\footnotetext{
${ }^{16}$ If $\mathbf{q}$ is fixed, the probability density of activation state $\mathbf{a}$ at thermal equilibrium is as follows (Chiang et al., 1987):

$$
p(\mathbf{a})=e^{H(\mathbf{a}) / T} / \int_{\mathbf{a}^{\prime}} e^{H\left(\mathbf{a}^{\prime}\right) / T} d \mathbf{a}^{\prime}
$$
}




\subsection{Incremental sentence comprehension in GSC}

In comprehension, the GSC model processes a length- $N_{\text {sent }}$ sentence $w_{1: N_{\text {sent }}}=w_{1} w_{2} \cdots w_{N_{\text {sent }}}$ word by word under a given commitment policy $\pi$ as follows. In each trial, the initial activation state of the model is set to $\mathbf{a}_{0} \sim N\left(\mathbf{a}_{0}^{*}, \boldsymbol{\sigma}_{\text {init }}^{2} \mathbf{I}\right)$ where $\mathbf{I}$ is the identity matrix and $\mathbf{a}_{0}^{*}$ is the global optimum when $\mathbf{q}=\mathbf{0}$. In all examples in Section $4, \sigma_{\text {init }}$ was set to 0.02 . The initial commitment state $\mathbf{q}_{0}$ is set to $\mathbf{0}$. For every word input $w_{k}$, the external input is updated discretely from $\mathbf{x}_{k-1}$ to $\mathbf{x}_{k}$ and the model updates $\mathbf{q}$ gradually from $\mathbf{q}_{k-1}$ to $\mathbf{q}_{k}$ under $\pi$. Because the Harmony surface changes (thus, the local optima shift) as a function of $\mathbf{q}$, when $\mathbf{q}$ changes, the activation state changes as well to climb up the dynamically changing Harmony surface (see Equation 6), from $\mathbf{a}_{k-1}$ to $\mathbf{a}_{k}$. After processing the last word $w_{N_{\text {sent }}}$, the external input is updated from $\mathbf{x}_{N_{\text {sent }}}$ to $\mathbf{x}_{N_{\text {sent }}+1}$ and the model initiates the wrap-up process. In the following, we describe how $\mathbf{x}$ and $\mathbf{q}$ are updated in more detail.

\subsubsection{Update of external input}

Let $\operatorname{cat}(w)$ be the set of all lexical categories that word $w$ can take. For example, consider lexical rules $\{N \rightarrow$ trains $\mid$ cats, $V \rightarrow$ trains $\mid$ sleeps $\}$. Then, cat(trains $)=\{\mathrm{N}, \mathrm{V}\}$ where $\mathrm{N}$ and $\mathrm{V}$ are members of the filler set $\mathscr{F}$. We denote the index set of a subset of symbols $\left\{A_{i}\right\} \subset \mathscr{A}$ as $I_{\mathscr{A}}\left(\left\{A_{i}\right\}\right)=\{i\}$.

Let $w_{k}$ be the $k$-th word of a sentence $w_{1: N_{\text {sent }}}$. For the input word $w_{k}$, we set $\mathbf{x}_{w_{k}}$ such that, in conceptual coordinates, its $(f, r)$-th component has the value of 2 if $f \in I_{\mathscr{F}}\left(\operatorname{cat}\left(w_{k}\right)\right)$ and $r$ is the index of role $(1, k)$ in $\mathscr{R}$, and has the value of 0 otherwise. Note that $(1, k)$ is the target terminal role associated with the $k$-th word input.

$\mathbf{x}_{w_{N_{\text {sent }}+1}}$, the input corresponding to the last word $w_{N_{\text {sent }}}$, is set in a special way. Simply speaking, for every irrelevant brick role given a length- $N_{\text {sent }}$ sentence, the input vector stimulates their target fillers (\#, @, or*@) directly. For example, if the input sentence is 'A B C' (see Figure 2b), $\mathbf{x}_{w_{4}}$ represents external input to bindings @/(1,4),*@/(2,3),*@/(3,2), and \#/(4,1). ${ }^{17}$

The actual external input vector $\mathbf{x}_{k}$ (which is different from $\mathbf{x}_{w_{k}}$ ) is defined as follows:

$$
\mathbf{x}_{k}=\lambda_{\mathrm{x}} \mathbf{x}_{k-1}+\mathbf{x}_{w_{k}}
$$

where $\mathbf{x}_{0}=\mathbf{0}$ and $\lambda_{\mathrm{x}}$ is the decay factor for the previous input $\left(0 \leq \lambda_{\mathrm{x}} \leq 1\right)$. If $\lambda_{\mathrm{x}}=1$, the model has perfect memory for all previous word inputs. If $\lambda_{\mathrm{x}}=0$, only the present word input $w_{k}$ is available to the model. In the present study, $\lambda_{\mathrm{x}}$ was set to 0.5 .

\subsubsection{Commitment policy}

A commitment policy specifies how the model updates $\mathbf{q}$. In a general form, $d \mathbf{q}=\pi(\mathbf{a}, \mathbf{q}, \mathbf{x}) d t$. Comprehenders likely learn this function $\pi$ in parallel with acquiring the grammar of a language. In the present study, we do not examine this issue, and deploy a fixed commitment policy.

\footnotetext{
${ }^{17}$ More formally, the $(f, r)$-th component of $\mathbf{x}_{w_{N_{\text {sent }}+1}}$ has a value of 2 (a) if $f \in I_{\mathscr{F}}(\{@\})$ and $r \in I_{\mathscr{R}}\left(\left\{(1, p) \mid N_{\text {sent }}<p \leq N_{\text {max }}, p \in\right.\right.$ $\mathbb{N}\}$ ); (b) if $f \in I_{\mathscr{F}}\left(\left\{{ }^{*} @\right\}\right)$ and $r \in I_{\mathscr{R}}\left(\left\{(l, p) \in \mathbb{N}^{2} \mid N_{\text {sent }}<l+p-1 \leq N_{\max }, 1<l<N_{\max }, 1<p<N_{\max }\right\}\right)$; (c) if $f \in I_{\mathscr{F}}(\{\#\})$ and $r \in I_{\mathscr{R}}\left(\left\{(l, 1) \mid N_{\text {sent }}<l \leq N_{\max } ; l \in \mathbb{N}\right\}\right)$; and has a value of 0 otherwise.
} 
For a GSC model constructed to deal with up to length- $N_{\max }$ sentences, define $\mathbf{t}=\left\{t_{1}, \cdots, t_{N_{\max }+1}\right\}$ as a vector of target $q$ values. As the model processes $w_{k}$, it updates $q_{r}$ where $r \in I_{\mathscr{R}}\left(\mathscr{R}_{k}\right)$, the components of $\mathbf{q}$ corresponding to brick roles in $\mathscr{R}_{k}=\{(l, p) \mid l+p=k+1\}$ - the set of brick roles aligned with $(1, k)$ in the diagonal line: $\mathscr{R}_{k}=\{(1, k),(2, k-1), \cdots,(k-1,2),(k, 1)\}$ (see Figure $2 \mathrm{a}$ ). These $q_{r}$ values are all equal, and increase from 0 to a value $t_{k}$ at the rate of $d q / d t=1$ and stay at the value $t_{k}$ until the following word $w_{k+1}$ is presented. After processing the last word of a sentence, the parser performs the wrap-up process during which the commitment strength of every brick role increases in the rate of $d q_{r} / d t=1$ until every component of $\mathbf{q}$ reaches $t_{N_{\max }+1}$.

Note that in the discussion below, we fix $d q / d t$ to 1 and allow $\mathbf{t}$ to vary. We therefore compactly define the policy for a grammar $\mathrm{G} \pi_{\mathrm{G}}$ as the vector $\mathbf{t}_{\mathrm{G}}$.

See Figure (17) for a visualization of this type of policy. Note the commitment strength of other brick roles (in the complement set of $\mathscr{R}_{k}$ ) does not change when the model processes $w_{k}$. This prevents the model from developing strong commitment to specific fillers in potentially irrelevant roles too early (for a schematic minimal example, see Figure 9); when the parser processes the first word of a sentence of unknown length, it is unlikely for the model to commit to a filler symbol as a potential 8-th word in brick role $(1,8)$.

Here, we set $\mathbf{t}$ to a constant value $q_{\max }$. This value must be chosen such that local optima of the model are close enough to grid points when $\mathbf{q}=q_{\max }$, such that the end product of incremental parsing is a discrete symbolic structure.

\subsection{Production in GSC}

We use the default wrap-up process to generate parse trees with no external input. In each trial, the initial activation state of the model is set to $\mathbf{a}_{0} \sim N\left(\mathbf{a}_{0}^{*}, \sigma_{\text {init }}^{2} \mathbf{I}\right)$ and the initial commitment level $\mathbf{q}$ is set to $\mathbf{0}$. With no external input, the model initiates the wrap-up process using the wrap-up policy $\pi_{0}$ until the minimal component of $\mathbf{q}$ reaches $q_{\max } .{ }^{18}$ Given a final activation state after the wrap-up process, its nearest grid point is taken as the parse tree that the model has generated in the trial. The probabilistic grammar that the GSC parser approximates can be estimated by iterating this generative process many times and computing relative frequencies of parse trees.

An important property of the GSC model is that it uses the same representation space and the same wrapup process for both production and comprehension. This is in line with the results of recent experimental studies (Kempen et al., 2012; Menenti et al., 2011; Segaert et al., 2012) and a modeling proposal (Kempen, 2014). In our model, comprehension is a special case of production under the influence of dynamically changing external input and a commitment policy specialized for comprehension.

\subsection{A heuristic training algorithm}

A Harmonic Grammar (HG) posits a (Grammar) Harmony value for each grammatical constraint expressed in an HG rule. By positing different Harmony values for different HG rules, it can naturally emulate prob-

\footnotetext{
${ }^{18}$ Preliminary investigation suggests that when the target grammar allows many grammatical constructions the wrap-up process encounters difficulty, producing some proportion of ungrammatical parse trees. This is an important area for future development of the model.
} 
abilistic context-free grammars. In standard, non-GSC probabilistic HG (also called "Maxent" grammar), only discrete combinatorial structures (corresponding to grid points) are considered, and the Harmony difference between two symbolic structures is assumed to be proportional to the difference of the log-probabilities of the two structures (Culbertson et al., 2013; Hayes and Wilson, 2008). In GSC modeling, however, this simple mapping between Harmony differences and probability ratios does not hold. For this reason, previous studies of GSC parsing either implemented a simple context-free grammar (e.g., Cho et al., 2017) or parametrically manipulated the Harmony value of a single HG rule while keeping the Harmony values of other HG rules to default values, introducing structural preference (Cho et al., 2018). In this section, we propose a heuristic algorithm that iteratively adjusts an HG (implemented in a GSC parser) to approximate a given PCFG.

Let $P(S)$ be the target probability distribution over complete (either grammatical or ungrammatical) parse trees $S_{i} \in \mathscr{S}$, the set of all complete parse trees. This distribution is either directly derived from the target probabilistic grammar or approximated by sampling sentences many times. Let $Q\left(S ; \mathbf{W}, \pi_{0}\right)$ be the probability of generating sentence $S$ using the GSC parser for production, described in Section 3.6. $\mathbf{W}\left(=\mathbf{W}_{\mathbf{c}}\right)$ contains the trainable parameters specifying the strength of grammatical constraints in conceptual coordinates. $\pi_{0}$ is the wrap-up policy that increases $q_{r}$ up to $q_{\max }$ at the rate $d q_{r} / d t=1$. (W and $\pi_{0}$ will sometimes be left implicit, and we will let $Q(\mathbf{v})$ denote $Q(S)$ when $\mathbf{v}$ is the vector representing $S$ and in the same contexts allow $\mathscr{V}$ to denote $\mathscr{S}$.) Because the model does not directly represent the probability distribution, it is estimated numerically by applying the wrap-up process to a set of initial GSC states many times. We evaluate the goodness of the model's approximation using the Kullback-Leibler (KL) divergence $D_{K L}$ of the GSC parser's probability distribution from the target distribution:

$$
J(\mathbf{W})=D_{K L}\left(P(S) \| Q\left(S ; \mathbf{W}, \pi_{0}\right)\right)=\sum_{i} P\left(S_{i}\right)\left(\log P\left(S_{i}\right)-\log Q\left(S_{i} ; \mathbf{W}, \pi_{0}\right)\right)
$$

At the end of the wrap-up process, $q_{r}=q_{\max }$ for every $r$ and every local optimum $\mathbf{a}^{*(i)}$ is close to a grid point $\mathbf{v}^{(i)}$ representing a parse tree $S_{i}$. If we treat each of these nearly discrete states as equivalent to the nearby grid point $\mathbf{v}$, the probability of each grid point $\mathbf{v}$ at thermal equilibrium can be approximated as follows (see Footnote 16):

$$
Q\left(\mathbf{v} ; \mathbf{W}, \mathbf{q}_{\max }\right) \approx \frac{e^{H(\mathbf{v}) / T}}{\sum_{\mathbf{v}^{\prime} \in \mathscr{V}} e^{H\left(\mathbf{v}^{\prime}\right) / T}}
$$

As $q_{r}$ increases, $Q\left(\mathbf{v} ; \mathbf{W}, \mathbf{q}_{\max }\right)$ approaches the above distribution (Tupper et al., 2014). ${ }^{19}$ Because $H(\mathbf{v})$ depends on $\mathbf{W}$, the strength of grammatical constraints, ${ }^{20}$ we get the following gradient (Ackley et al., 1985):

$$
\frac{\partial J(\mathbf{W})}{\partial w_{f r, f^{\prime} r^{\prime}}}=\sum_{i} \frac{\partial J(\mathbf{W})}{\partial H_{G}\left(\mathbf{v}^{(i)}\right)} \frac{\partial H_{G}\left(\mathbf{v}^{(i)}\right)}{\partial w_{f r, f^{\prime} r^{\prime}}} \approx \sum_{i}\left\{\frac{1}{T}\left(P\left(S_{i}\right)-Q\left(S_{i}\right)\right)\right\}\left\{\frac{1}{2} v_{f r}^{(i)} v_{f^{\prime} r^{\prime}}^{(i)}\right\}
$$

where $\mathbf{v}$ is a grid point in conceptual coordinates and $H_{G}(\mathbf{v})=(1 / 2) \mathbf{v}^{\top} \mathbf{W}_{\mathbf{c}} \mathbf{v}$ (see Eq. 4). ${ }^{21}$ In our case,

\footnotetext{
${ }^{19}$ As $q \rightarrow \infty, \mathbf{a}^{*(i)} \rightarrow \mathbf{v}^{(i)}$ and the probability mass is concentrated on the grid points.

${ }^{20}$ Every grid point has the same values of $H_{B}, H_{R}$, and $H_{D}$.

${ }^{21}$ In production trials, the external input vector $\mathbf{x}_{\mathbf{c}}$ in conceptual coordinates is fixed at $\mathbf{0}$. Thus, the Grammar Harmony of a grid point $\mathbf{v}$ with respect to the external input is $0: \mathbf{x}_{\mathbf{c}}\left(\mathbf{N}^{\top} \mathbf{N}\right) \mathbf{v}=0$.
} 
$Q(\mathbf{v})$ may not approximate the expected Boltzmann distribution well because the system does not reach thermal equilibrium in each trial. However, it is still expected that the equation above changes the parameter values in a direction that reduces our approximation cost $J(\mathbf{W})$. This is because the activation state space is continuous so enhancing the Grammar Harmony of a grid point representing a parse tree will enhance the Grammar Harmony of intermediate activation states in proportion to how similar they are to the grid point. Thus, during the wrap-up process, the model is more likely to move to the grid point. Because the scaling factor $P\left(S_{i}\right)-Q\left(S_{i}\right)$ is only estimated approximately, we treat this algorithm as heuristic; below, we investigate how the algorithm works numerically.

As a concrete example of the weight update procedure, consider a simple probabilistic grammar that generates two grammatical structures NP[1] $\left(\right.$ Det, $\left.\mathrm{N}_{\mathrm{sg}}\right)$ (e.g., "the $\operatorname{dog}$ ") and NP[2](Det, $\left.\mathrm{N}_{\mathrm{pl}}\right)$ (e.g., "the dogs") equally frequently, where $\mathrm{N}_{\mathrm{sg}}$ and $\mathrm{N}_{\mathrm{pl}}$ denote singular and plural noun categories, respectively. Figure 10 presents a graph of grammatical constraints that collectively approximate the target grammar; only a subset of constraints and bindings is presented for simplicity. In each iteration, the algorithm generates symbolic structures via the wrap-up process many times, say 100 times. Suppose two grammatical structures S[1] $\equiv \mathrm{NP}[1]\left(\right.$ Det, $\left.\mathrm{N}_{\mathrm{sg}}\right)$ and $\mathrm{S}[2] \equiv \mathrm{NP}[2]\left(\right.$ Det, $\left.\mathrm{N}_{\mathrm{pl}}\right)$ were produced 20 times and 30 times, respectively. An ungrammatical structure, S[3], where 'the dog' is parsed as a plural noun phrase NP[2] (NP[2](Det, $\left.\mathrm{N}_{\mathrm{sg}}\right)$ ) was produced 50 times. No other ungrammatical structures (e.g., Det(Det,Det)) were produced. Ignoring all unproduced, ungrammatical structures, the algorithm considers two distributions $P(S)=[0.5,0.5,0]$ and $Q(S)=[0.2,0.3,0.5]$ across three structures $\mathrm{S}[1], \mathrm{S}[2]$, and S[3]. Given the observed distribution $Q(S)$, the algorithm needs to increase $Q(S[1])$ and $Q(S[2])$ but to decrease $Q(S[3])$. Consider how the algorithm can decrease $Q(S[3])$. S[3] consists of bindings Det/(1,1), $\mathrm{N}_{\mathrm{sg}} /(1,2)$, and NP[2]/(2,1) thus $H_{G}(S[3])=$ $H_{G}(\operatorname{Det} /(1,1), \mathrm{NP}[2] /(2,1))+H_{G}\left(\mathrm{~N}_{\mathrm{sg}} /(1,2), \mathrm{NP}[2] /(2,1)\right)+H_{G}(\operatorname{Det} /(1,1))+H_{G}\left(\mathrm{~N}_{\mathrm{sg}} /(1,2)\right)+H_{G}(\mathrm{NP}[2] /(2,1))$. Following the values in Figure 10, $H_{G}(S[3])=w_{15}+w_{25}+b_{1}+b_{2}+b_{5}$. To reduce the Grammar Harmony of $\mathrm{S} 3$, each of these five grammatical parameter values is reduced: for example, $\Delta w_{15} \propto P(S[3])-Q(S[3])=$ -0.5 . Note that the parameter $w_{15}$ contributes to the Grammar Harmony of S[2] as well; $\Delta w_{15} \propto P(S[2])-$ $Q(S[2])=0.2$. If we consider all structures, then $\Delta w_{15} \propto \sum_{i=1}^{3}(P(S[i])-Q(S[i]))=-0.3$. A similar procedure is used to update all other $w \mathrm{~s}$ and $b \mathrm{~s}$.

One practical issue is how to estimate $Q(S)$ reliably without running a huge number of trials. Such sampling is computationally expensive, as each production trial requires updating the activation state and the commitment state many times. Instead of reliably estimating $Q(S)$ every iteration, we use a relatively small number of trials to get $\tilde{Q}(S)$, a noisy estimate of $Q(S)$, and take its exponential moving average as $Q(S): Q\left(S_{i}\right)(t)=\left(1-\lambda_{\mathrm{Q}}\right) \tilde{Q}\left(S_{i}\right)(t)+\lambda_{\mathrm{Q}} Q\left(S_{i}\right)(t-1)(t>1), Q\left(S_{i}\right)(1)=\tilde{Q}\left(S_{i}\right)(1)$, where $t$ is the iteration number and $\tilde{Q}\left(S_{i}\right)$ is the relative frequency of $S_{i}$ observed in the sample from $t$-th iteration.

\section{Examples}

A major motivation underlying the proposed architecture is potential scalability, but at this point there are practical challenges in building and investigating a large-scale GSC model which can parse many multi-word linguistic constructions. Because of the computational cost of training a model with a large number of pro- 


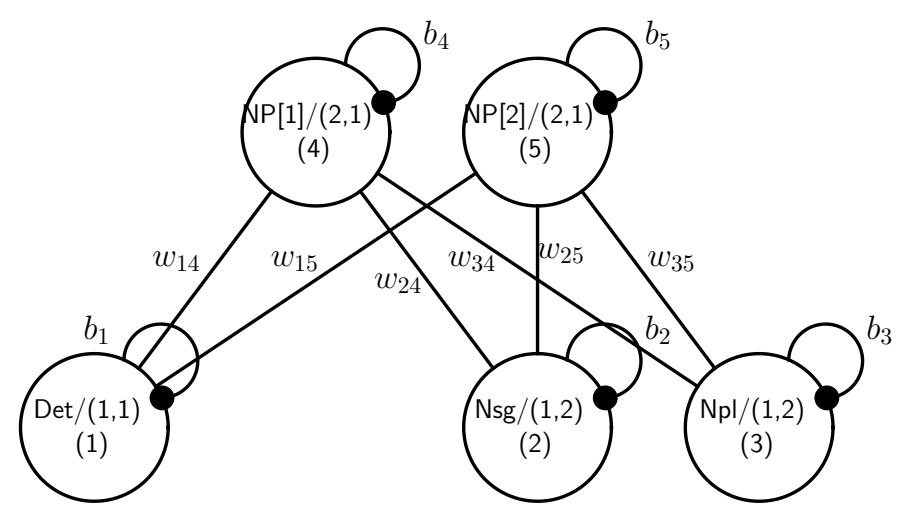

Figure 10: A graph of (a subset of) grammatical constraints ( $w$ 's and $b$ 's) that, after training, approximate a simple probabilistic grammar, which generates two structures $\mathrm{NP}[1]\left(\right.$ Det, $\left.\mathrm{N}_{\mathrm{sg}}\right)$ and $\mathrm{NP}[2]\left(\right.$ Det, $\left.\mathrm{N}_{\mathrm{pl}}\right)$ equally frequently.

cessing units, in this section we consider two simplified grammars and implement a separate GSC model for each. In both examples, we chose expansion probabilities for non-terminal symbols arbitrarily and did not model lexical rules. Our goal here is not to model particular human linguistic behavior (comprehension or production), but to demonstrate how a continuous dynamical system model can compute (representations of) well-formed structures gradually, and manage the structural uncertainty arising in incremental processing. The example mini-grammars were selected because they generate psycholinguistically interesting sentences, setting the stage for future work modeling psycholinguistic data.

Each subsection is organized in the following way. We begin with a set of rewrite rules. These rewrite rules may not follow typical linguistic analyses; for example, in Grammar 1, N and Vi combine directly to form the $\mathrm{S}$ constituent without a VP. Here we take a grammar as specifying which two constituents can be merged to form a new constituent (with an associated probability). We report the model size (the numbers of fillers, roles, and processing units [equal to the number of bindings]) and the training result in terms of KL divergence, production accuracy, and the parser's internal model of the language's probability distribution over sentences, $Q(S)$. Then, we report incremental parsing accuracy under multiple, manually chosen commitment policies.

The general training regime is as follows: the learning rate was set to 0.1 . The decay parameter $\lambda_{\mathrm{Q}}$ for $Q(S)$ was set to 0.04 . The estimate of $Q(S), \tilde{Q}(S)$, was obtained from 4 production trials. Note that these parameters caused the estimate to fluctuate even after many learning steps. If the fluctuation holds within a reasonable range near the target probabilities, we take it as weak evidence for convergence under the training regime. In all cases, the following parameter values were used: initial commitment state $\mathbf{q}_{0}=\mathbf{0}$, the maximum commitment level $q_{\max }=15$, input decay rate $\lambda_{\mathrm{x}}=0.5$, resource constraint coefficient $m=$ 30, computational temperature $T=0.01$, time step constant $\Delta t=0.005$ used in Equation 7 for numerical integration of Equation 6. 


\subsection{Grammar 1}

We characterize Grammar 1 (G1) as the following set of rewrite rules, where the pipe symbols separate alternative expansions and the numbers in parentheses denote expansion probabilities.

- $\mathrm{S} \rightarrow(0.35) \mathrm{N}$ Vi $\mid(0.60) \mathrm{N}$ VP $\mid(0.05) \mathrm{NP} \mathrm{Vi}$

- $\mathrm{NP} \rightarrow(1.0) \mathrm{N} \mathrm{RC}$

- $\mathrm{RC} \rightarrow(1.0) \mathrm{Vpp} \mathrm{PP}$

- VP $\rightarrow(0.5)$ Vi PP $\mid$ (0.3) BE Vpp | (0.2) BE VPpp

- VPpp $\rightarrow$ (1.0) Vpp PP

- $\mathrm{PP} \rightarrow(1.0) \mathrm{P} \mathrm{N}$

G1 generates 5 sentence types of 5 words long or less (sentence probability in parentheses):

S[1] (.35) "The-horse (N) fell (Vi)"

$\mathrm{S}$ 2] (.30) "The-horse $(\mathrm{N})$ ran $(\mathrm{Vi})$ past $(\mathrm{P})$ the-barn $(\mathrm{N})$ "

$\mathrm{S}$ [3] (.18) "The-horse (N) was (BE) raced (Vpp)"

$\mathrm{S}$ [4] (.12) "The-horse (N) was (BE) raced (Vpp) past (P) the-barn (N)"

$\mathrm{S}[5](.05)$ "The-horse(N) $\operatorname{raced}(\mathrm{Vpp}) \operatorname{past}(\mathrm{P})$ the-barn(N) fell(Vi)"

Note that S[5] corresponds to the classic example of a garden-path sentence. A GSC model consisting of 405 processing units (27 fillers x 15 roles) was constructed and trained to approximate G1.

The training result is presented in Figure 11. Overall, the model approximates the target distribution well. The mean KL divergence over the last 100 updates (corresponding to 400 production trials) was 0.022 $(S D=0.006) ; P(S)=[0.35,0.3,0.18,0.12,0.05]$ and $Q(S)=[0.349,0.295,0.179,0.116,0.047]$. The mean production accuracy was $0.987(S D=0.005)$.

Figure 12 presents parsing accuracy as a function of commitment policies. To reduce the search space, we constrained the policies by setting each element of the target-commitment-level vector $\mathbf{t}_{\mathrm{G} 1}$ to a constant value $t$. Parsing accuracy was 1 when $t \in\{4,5,6\} .22$

Figure 13 presents a sample trajectory of activation states in the 405-dimensional state space as the model processed a sentence ' $\mathrm{N}$ Vi P N' under commitment policy $\pi_{\mathrm{G} 1}$ with $\mathbf{t}_{\mathrm{G} 1}=(4,4,4,4,4)$. The distributed activation state change in the processing units, in neural coordinates (in which the simulation is carried out), is shown in the upper panel. The state trajectory is symbolically interpretable via the change of basis to conceptual coordinates, shown in the lower panel. Figure 14 reveals the underlying state change

\footnotetext{
${ }^{22}$ Note that the policy space presented in Section 3.5.2 is much greater than the policy space investigated here. The target commitment value $t_{k}$ for the $k$-th word input may change depending on the position $k$. In fact, we can consider a wider policy space in which $t_{k}$ depends not on the position but on the prefix $w_{1: k}$. An important question is what commitment policy explains human experimental data well. We reserve the question for future studies and focus on demonstrating the model's ability to parse sentences accurately.
} 

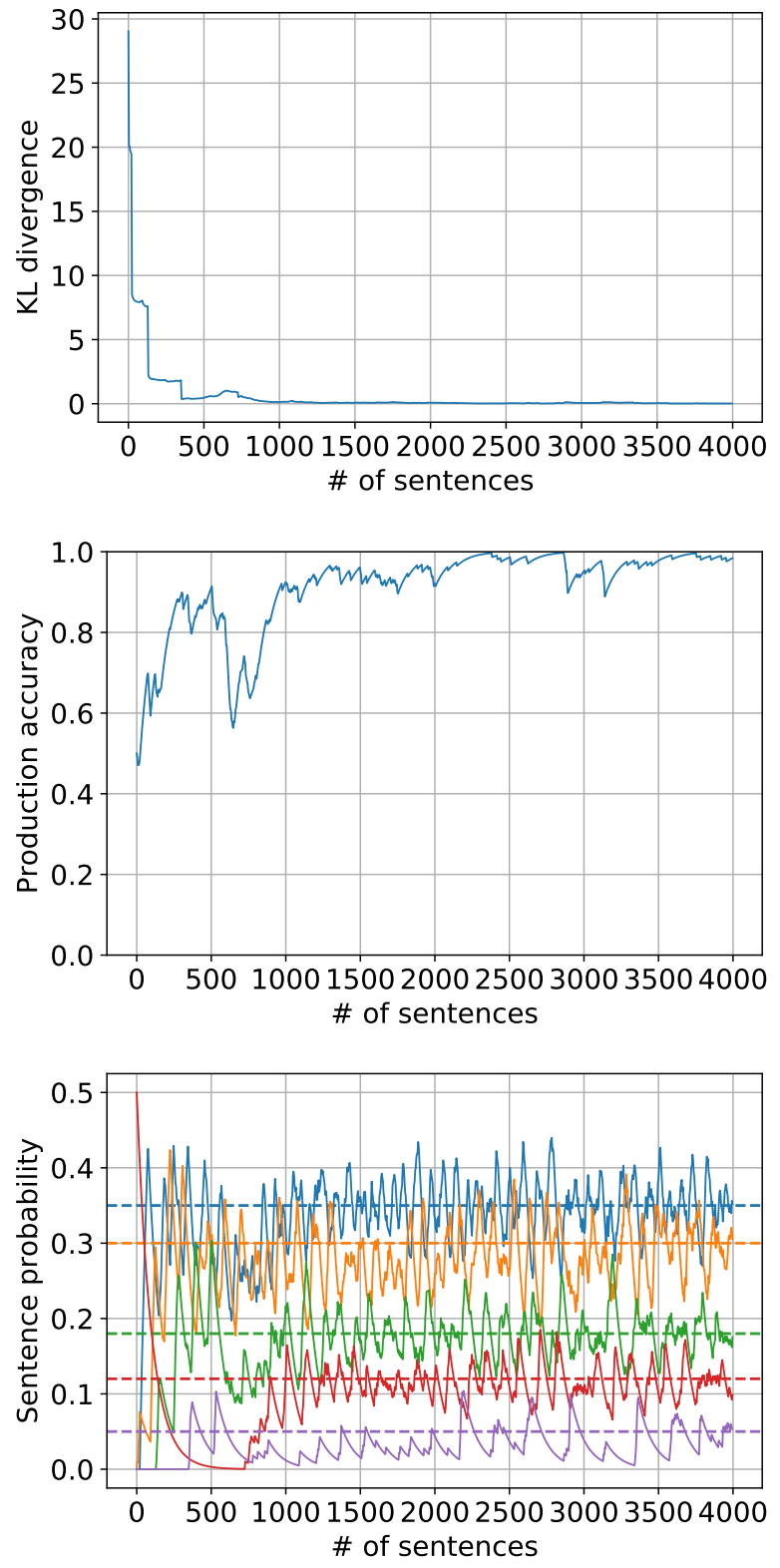

Figure 11: G1 training result. (Top) Change in KL divergence between $P(S)$ and $Q\left(S \mid \mathbf{W}, \pi_{0}\right)$ over the number of sentences produced. (Middle) The proportion of grammatical structures (or grid points) in production (or production accuracy). (Bottom) Estimated language model $Q\left(S \mid \mathbf{W}, \pi_{0}\right)$. The horizontal dashed lines indicate target probabilities for different sentence types. 


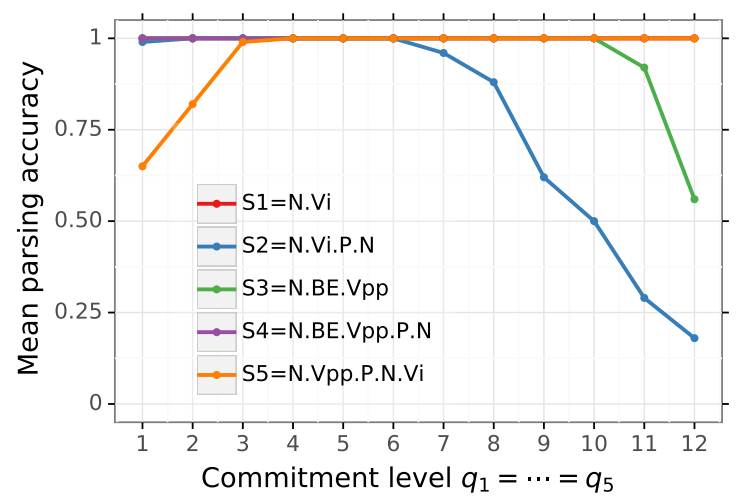

Figure 12: Parsing accuracy per sentence type under different commitment policies for grammar G1.

more clearly by grouping and arranging binding activations according to structural roles. The labels in each brick role present the top two most active fillers in the role over the course of processing. As the model processes the input sentence word by word, it gradually reduces the future possibilities by committing to some filler symbols in different brick roles.

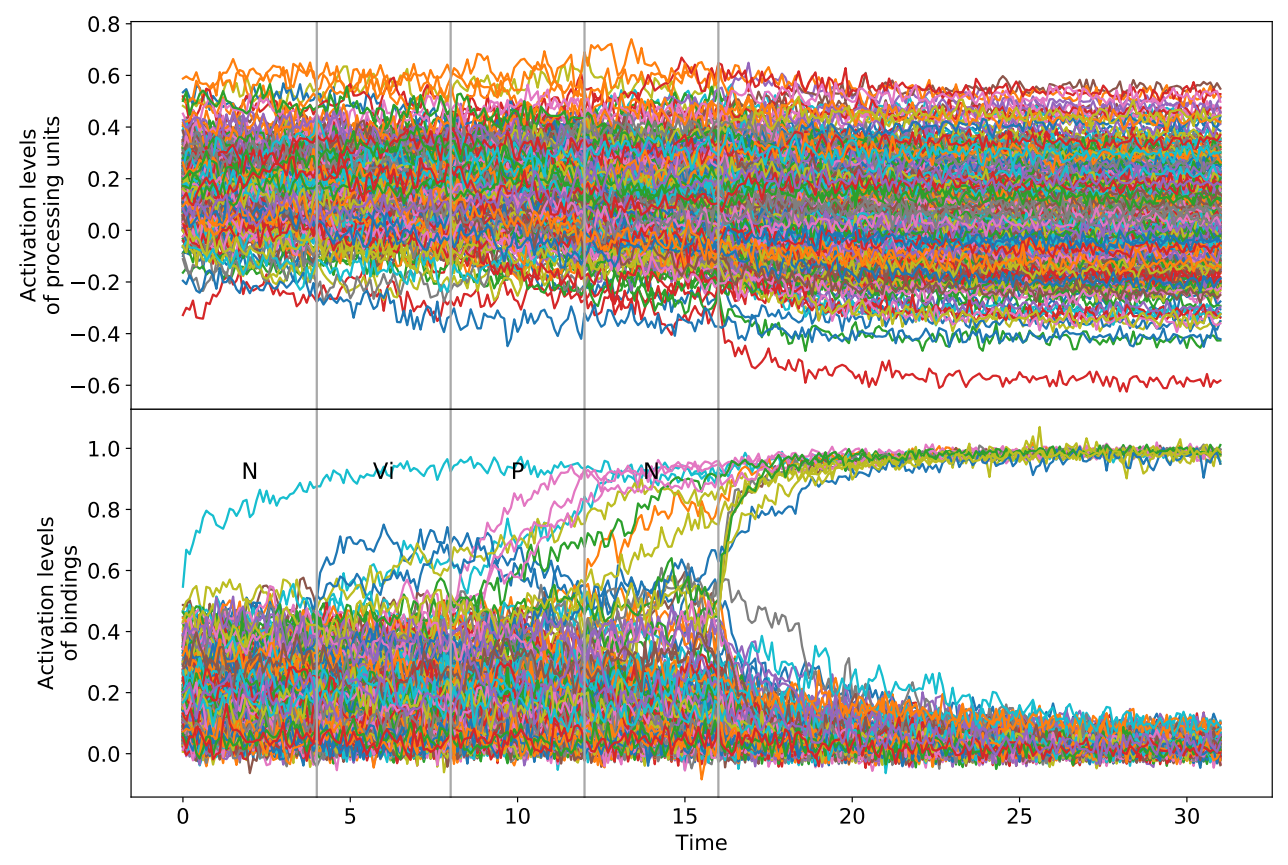

Figure 13: Trajectories of activation states in two different coordinates. (Top: neural coordinates) the activation levels of processing units. (Bottom: conceptual coordinates) the activation levels of filler/role bindings.

The intermediate activation states at three different time points during processing of $\mathrm{N}$ Vi $\mathrm{P} \mathrm{N}$ are presented in Figure 15. For notational convenience, we introduce the following conventions: $\mathrm{X}_{(l, p)}^{\prime k} \equiv \mathrm{X}[k]$ : $0 /(l, p)$ and ' $\hat{\mathrm{Y}}_{(l, p)}^{k} \equiv * \mathrm{Y}[k]: 1 /(l, p)$. The prime and backprime symbols denote two context-free roles 0 and 1, respectively, where $\mathrm{X}: 0$ denotes $\mathrm{X}$ as the first child of its parent and $\mathrm{X}: 1$ denoting the second child of 




Figure 14: Trajectories of filler/role binding activations in conceptual coordinates, grouped and arranged to make patterns clear. (n.b. :0 and :1 denote left- and right-child symbols; see Section 3.1.1.) The shaded regions correspond to the time intervals during which the model processed each word. The commitment strength assigned to each brick role was updated in the interval and in the wrap-up process. Each panel contains two labels of fillers that were most active (top > bottom) over the course of time in each brick role. 
its parent. The superscript denotes an alternate form of the same filler symbol. The hat symbol denotes that the symbol is a copy symbol. Finally, the subscript denotes the brick role that binds the filler symbol. After processing the second word $\mathrm{Vi}, \mathrm{Vi}^{\prime}$ and ' $\mathrm{Vi}$ were more strongly activated than other fillers in role $(1,2)$ and $\hat{\mathrm{N}}^{\prime}$ and $\mathrm{S}^{\prime 1}$ were more strongly activated than others in role $(2,1)$. The partial activations of $\mathrm{N}_{(1,1)}^{\prime},{ }^{\prime} \mathrm{Vi}_{(1,2)}$, and $\mathrm{S}_{(2,1)}^{\prime 1}$ suggest that the model considers $\mathrm{S}_{(2,1)}^{\prime 1}\left(\mathrm{~N}_{(1,1)}^{\prime}, \mathrm{V}_{(1,2)}\right)$ as a candidate interpretation. The partial activations of $\mathrm{N}_{(1,1)}^{\prime}, \mathrm{Vi}_{(1,2)}^{\prime}, \hat{\mathrm{N}}_{(2,1)}^{\prime}$ suggest that the model predicts alternative interpretations consistent with $?_{1}\left(\mathrm{~N}, ?_{2}\left(\mathrm{Vi}, ?_{3}\right)\right)$, e.g., $\mathrm{S}_{(4,1)}^{\prime 2}\left(\mathrm{~N}_{(1,1)}^{\prime}, \mathrm{VP}_{(3,2)}^{1}\left(\mathrm{Vi}_{(1,2)}^{\prime},{ }^{\prime} \mathrm{PP}_{(2,3)}^{1}\left(\mathrm{P}_{(1,3)}^{\prime}, \mathrm{N}_{(1,4)}\right)\right)\right.$ (omitting copy symbols). If the second word is the final word of the sentence, the model will initiate the wrap-up process to drop out the latter interpretations and choose the first interpretation. If the second word is followed by another word, the first interpretation will be eventually dropped out. In this case, there was a third word input. After processing the third word, the model developed a rather strong expectation for the lexical category of the following word ' $\mathrm{N}_{(1,4)}$ and non-terminal symbols ' $\mathrm{PP}_{(2,3)}^{1}$ and ${ }^{\prime} \mathrm{VP}_{(2,2)}^{1}$ (see the left bottom panel of Figure 15). This example suggests that the model has the ability of maintaining multiple possibilities and expecting likely continuations/structures based on what it has processed. The right bottom panel presents the parse tree constructed from the final activation state after the wrap-up process, showing that the model parsed the input sentence accurately.

Instead of individual binding activations, the activation levels of treelets at a particular role can be investigated. We define the activation level of a treelet at role $(l, p)$ as the average activation level of its constituent bindings. For example, the activation of treelet $\mathrm{S}^{1}\left(\mathrm{~N}^{\prime},{ }^{\prime} \mathrm{Vi}\right)$ at role $(2,1)$ is defined as the average activation of bindings $\mathrm{S}_{(2,1)}^{\prime 1}, \mathrm{~N}_{(1,1)}^{\prime}$, and ${ }^{\prime} \mathrm{Vi}_{(1,2)}$. The activation of treelet $\hat{\mathrm{N}}^{\prime}\left(\mathrm{N}^{\prime}\right.$, ), with no right child. at role $(2,1)$ is defined as the average activation of bindings $\hat{\mathrm{N}}_{(2,1)}^{\prime}$ and $\mathrm{N}_{(1,1)}^{\prime}$; this unary-branching treelet is allowed by the copy rule $\hat{\mathrm{N}} \rightarrow \mathrm{N}$. Figure 16 presents the activation trajectories of the four most active treelets at roles $(2,1)$ and $(3,2)$ as the model processed the sentence ' $\mathrm{N}$ Vi P N'; the four most active ones out of all grammatical treelets are presented. Any blend state can be viewed as a conjunctive blend of partially active treelets some of which may conflict with each other. (Note that due to representational overlap between treelets, at the end of processing, the activations of the 3 rejected treelets do not fall to 0 .)

Before closing this example, we demonstrate a unique property of the GSC model as opposed to alternative connectionist models of sentence comprehension. The GSC model can maintain multiple analysis possibilities (see Section 2.3) under intrinsic update noise by freezing the commitment levels. So far we considered the self-paced reading situation in which the model controlled the timing of the external input (the $(k+1)$-th word was presented after the model had reached $q_{k}$ ). Now we consider an analogue to the comprehension of spoken sentences, where the comprehender does not control the timing of the external input; each word is presented for a fixed amount of time (15 [arbitrary] time units). For example, as the model processed the first word input under the same commitment policy $\pi_{\mathrm{G} 1}$, the commitment level in brick role $(1,1)$ increased from 0 to 4 for 4 time units and then stayed at 4 for additional 11 time units before the input was updated. As shown in Figure 17, when external input and commitment levels are fixed, the location of local optima in the state space (i.e., conjunctive blends) are fixed as well. The GSC model moves to a nearby optimum and stays in a neighborhood of this local optimum, showing that parallel parsing is dynamically stable. 

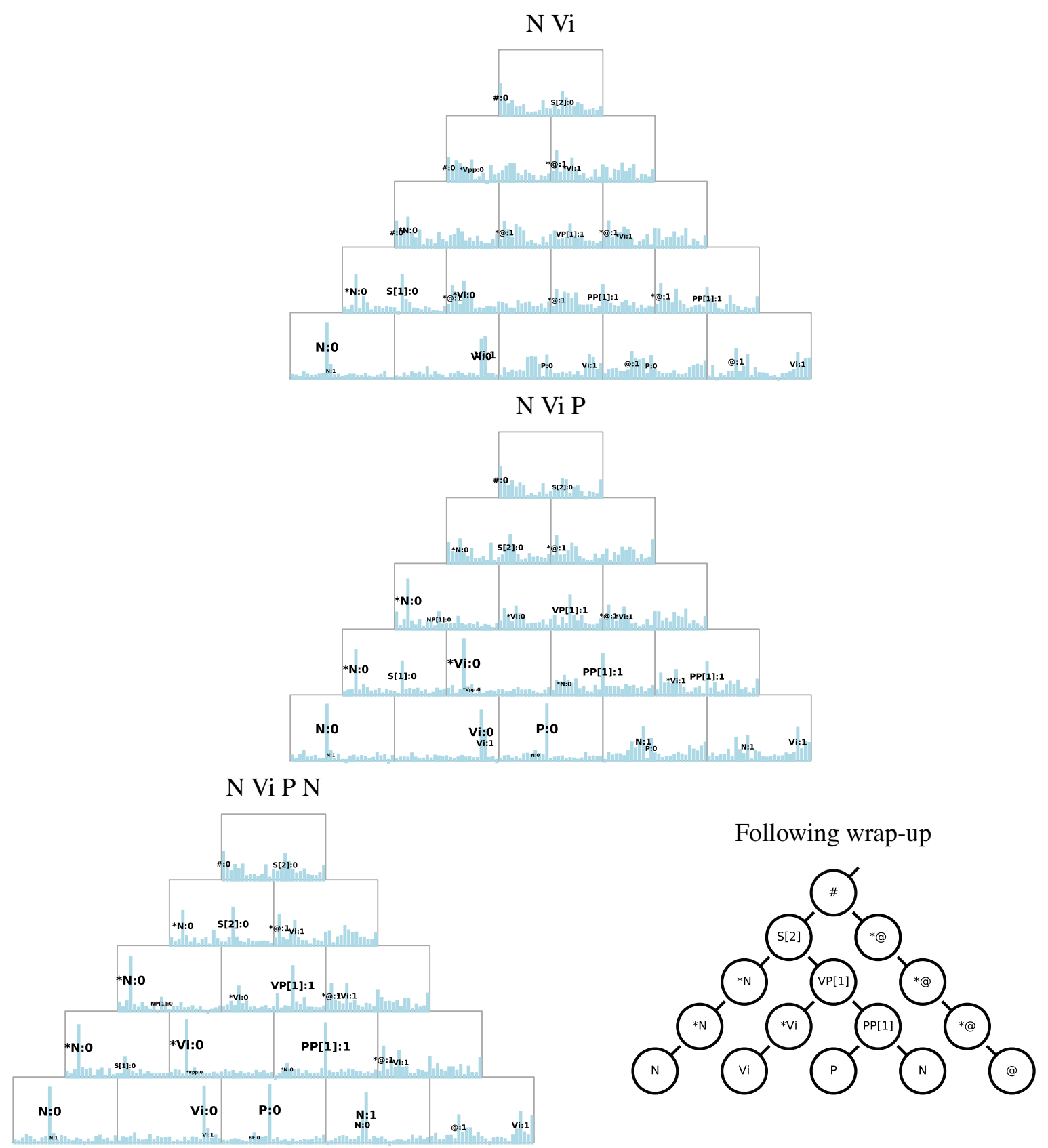

Figure 15: Activation states after processing the second (top), the third (middle), the fourth (bottom left) word of sentence ' $\mathrm{N}$ Vi P N'. The heights of bars in each brick role represent the activation levels of fillers. Names of the two most active fillers in each brick role are presented; font sizes are proportional to activation levels. The right bottom panel presents the parse tree reconstructed from the final activation state after the wrap-up process. 

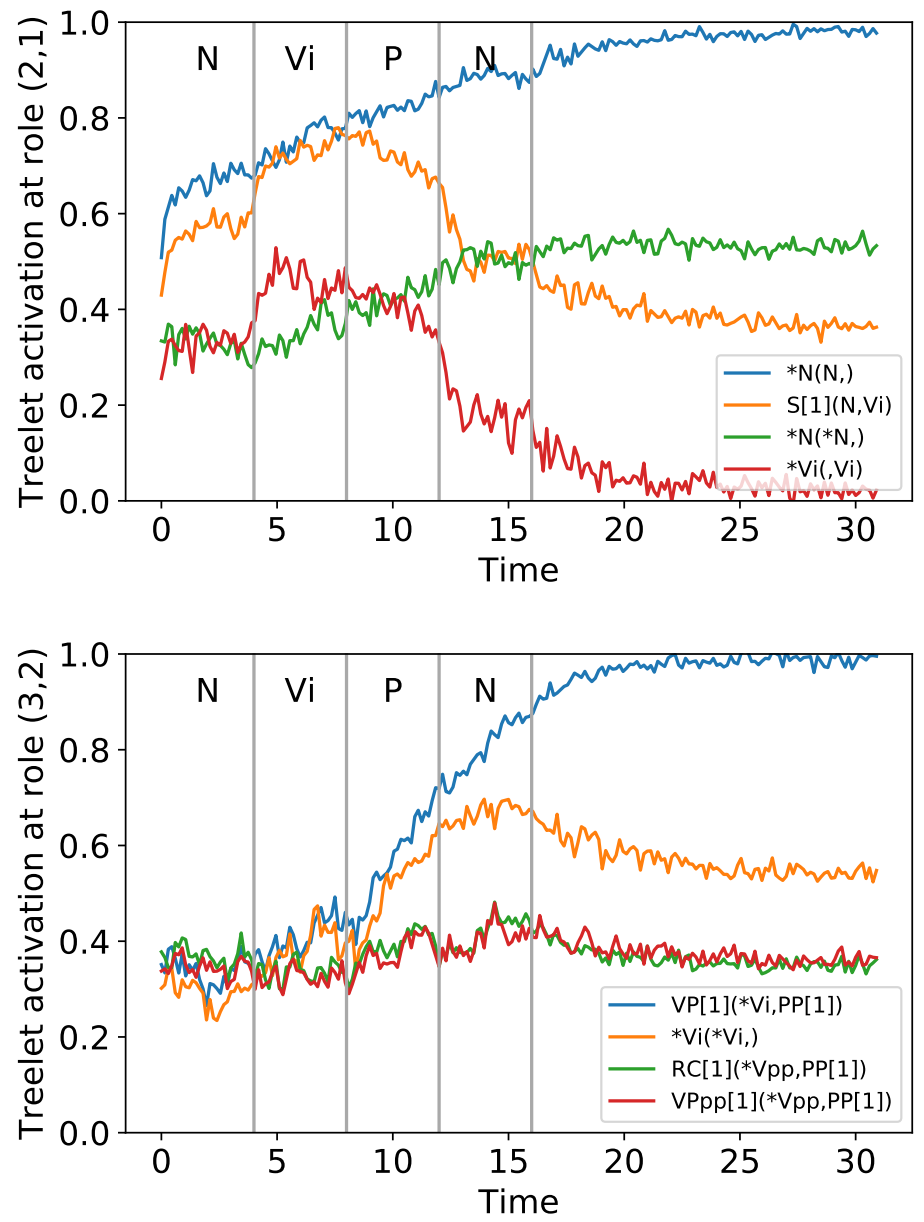

Figure 16: Trajectories of treelet activations (see text for definition) at role $(2,1)$ (top) and role $(3,2)$ (bottom). For example, $\mathrm{S}[1](\mathrm{N}, \mathrm{Vi})$ at role $(2,1)$ represents the average activation of $\{\mathrm{S}[1]: 0 /(2,1), \mathrm{N}: 0 /(1,1)$, $\mathrm{Vi}: 1 /(1,2)\}$. In each panel, we present the four most active grammatical treelets in each brick role. (Note position-specific role ' 0 ' and ' 1 ' is omitted for clarity.) 


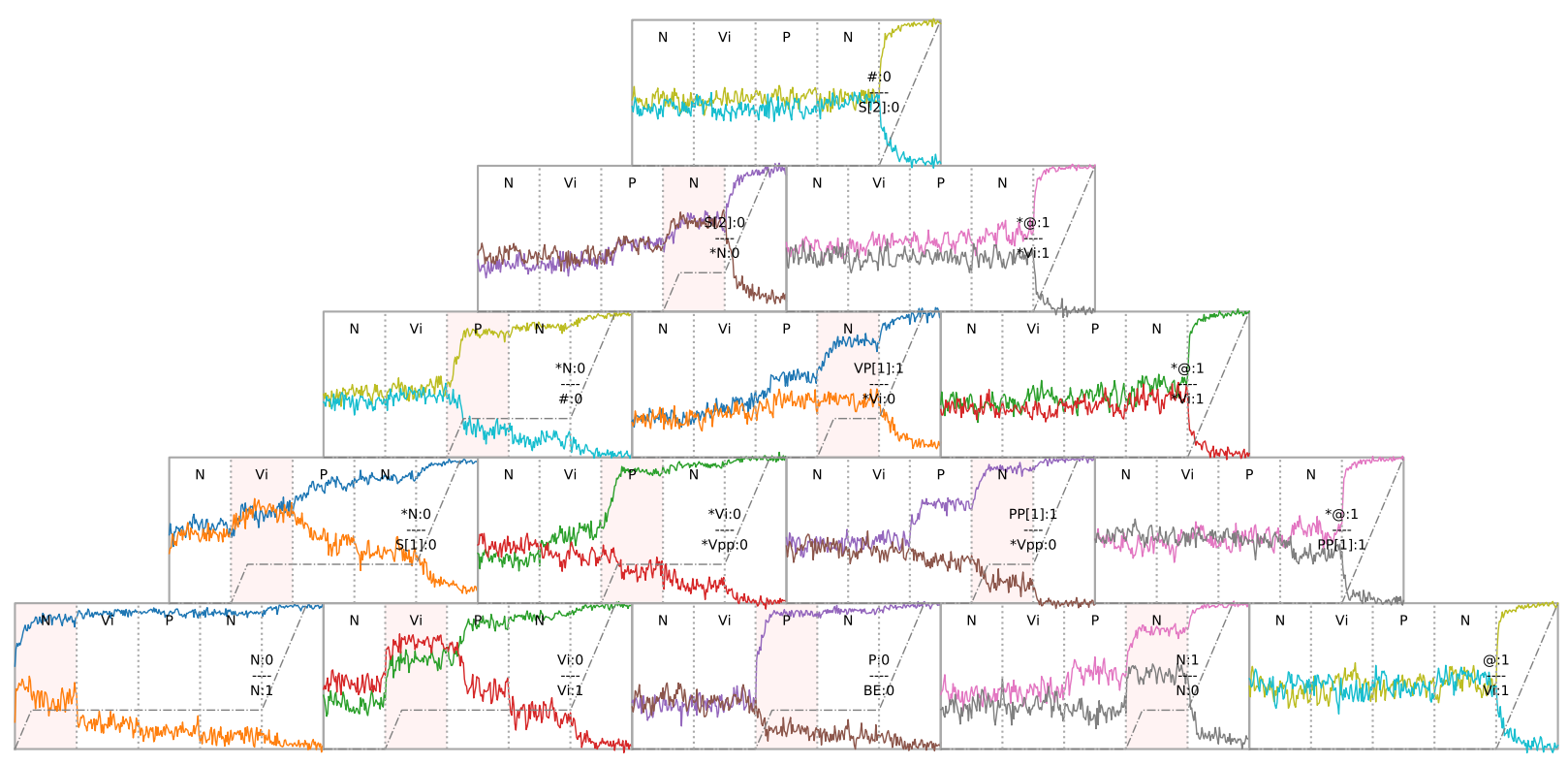

Figure 17: Trajectories of activation states of two most active fillers in each brick role as the model processed a sentence ' $\mathrm{N}$ Vi P N' under the same commitment policy as above $\pi_{\mathrm{G}_{1}}$, but slowing down the presentation of each word to 15 time units instead of 4 . The gray dashed line in each brick role presents the (normalized) commitment strength in the role. Where this line is horizontal, between two vertical dotted lines (during the processing of a single word) the external input and commitment level are constant, and the curves show sustained, stable activation of the optimal blend. 


\subsection{Grammar 2}

The parser's good performance extends to other relatively complex grammars. Here we examine a grammar with embedding, varying levels of probability for grammatical sentences, and an ambiguous lexical item.

- $\mathrm{S} \rightarrow(0.6) \mathrm{N} \mathrm{VP} \mid(0.4) \mathrm{NP} \mathrm{VP}$

- $\mathrm{NP} \rightarrow(0.8) \mathrm{N}$ RCs $\mid(0.2) \mathrm{N}$ RCo

- $\mathrm{RCs} \rightarrow(1.0) \mathrm{RPs} \mathrm{VP}$

- $\mathrm{RCo} \rightarrow(1.0) \mathrm{RPo} \mathrm{NV}$

- $\mathrm{NV} \rightarrow(1.0) \mathrm{N} \mathrm{Vt}$

- $\mathrm{VP} \rightarrow(1.0) \mathrm{Vt} \mathrm{N}$

Grammar G2 generates 3 sentence types of 6 words long or less:

$\mathrm{S}$ [1] (.60) "The-reporter" (N) "attacked" (Vt) "the-senator" (N)

$\mathrm{S}$ 2] (.32) "The-reporter" (N) "that" (RPs) "attacked" (Vt) "the-senator" (N) "admitted" (Vt) "the-error" $(\mathrm{N})$

$\mathrm{S}$ [3] (.08) "The-reporter" (N) "that" (RPo) "the-senator" $(\mathrm{N})$ "attacked" (Vt) "admitted" (Vt) "the-error" $(\mathrm{N})$

Note S[2] and S[3] reflect the well-studied contrast between Subject (SRC) and Object Relative Clause (ORC) sentences (King and Just, 1991). A GSC model consisting of 504 processing units (24 fillers x 21 roles) was constructed and trained to approximate G2. Note G2's lexical ambiguity: RPs and RPo have the

same word form (e.g., 'that'). In our parsing simulations, both bindings $\operatorname{RPs}_{(1,2)}^{\prime}$ and $\operatorname{RPo}_{(1,2)}^{\prime}$ received the same external input as the model was processing the second word of S[2] and S[3].

The training result is presented in Figure 18. Overall, the model seems to approximate the target distribution well. The mean KL divergence over the last 100 updates (corresponding to 400 production trials) was $0.008(S D=0.003) ; P(S)=[0.6,0.32,0.08]$ and $Q(S)=[0.595,0.319,0.080]$. The mean production accuracy was $0.994(S D=0.003)$. Figure 19 presents parsing accuracy as a function of commitment policies. Parsing accuracy was 1 when $t \in\{6, \cdots, 12\}$. This provides additional evidence that our model can handle embedded structures - even when one structure (ORC) is far less frequent than another (SRC) and there is lexical ambiguity in the initial word of each embedded clause.

\section{General Discussion}

In this study, we proposed a continuous-time, continuous-state stochastic dynamical system model of online sentence comprehension and production, extending related previous work (Cho et al., 2018, 2017) to more general cases. This approach allows us to provide answers to the three key computational questions raised in the introduction: 



Figure 18: G2 training result. (Top) KL divergence of $P(S)$ and $Q\left(S \mid \mathbf{W}, \pi_{0}\right)$ over the number of sentences produced. (Middle) The proportion of grammatical structures (or grid points) in production (or production accuracy). (Right) Estimated language model $Q\left(S \mid \mathbf{W}, \pi_{0}\right)$. The horizontal dashed lines indicate target probabilities for different sentences. 


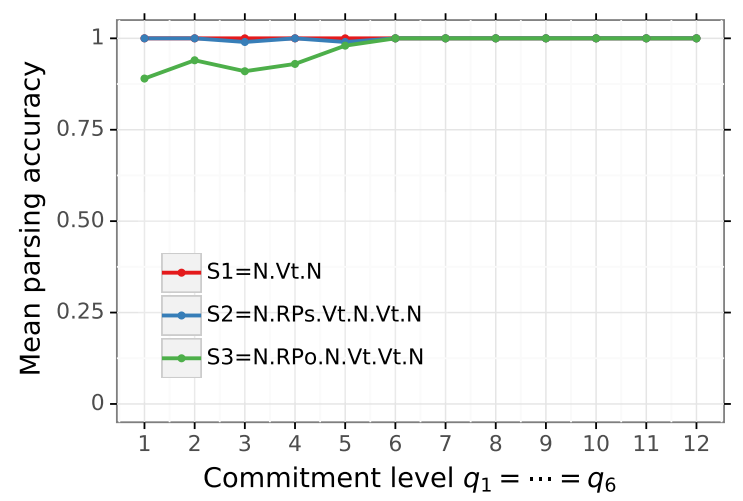

Figure 19: Parsing accuracy per sentence type under different commitment policies for grammar G2.

(A1) The mapping between a discrete set of symbolic structures and a continuous state space: Tensor product variable binding provides the GSC parser with a general, lossless encoding procedure for mapping a discrete symbolic structure, expressed in terms of filler/role bindings, to a distributed activation vector.

(A2) The mapping between a continuous state space and a set of probability distributions: Via dynamic unblending, the GSC parser probabilistically maps conjunctive blends of the vectors representing multiple symbolic constituents to vectors of activation representing discrete symbolic structures.

(A3) The stability of the representation of multiple parses in the continuous state space: Dynamic unblending is governed by a control parameter: the commitment level. Until the commitment state or external input changes, the parser's activation state will remain in a stable conjunctive blend state.

This last point - the use of a commitment policy to dynamically manage structural uncertainty during incremental parsing — is the key feature distinguishing the GSC model from other dynamical cognitive approaches (see beim Graben and Hutt, 2015; Hutt and beim Graben, 2017 for a related neural approach). This allows the GSC parser to be a truly parallel parser: multiple analysis possibilities can be robustly maintained across words in a principled way. In contrast, alternative dynamic neural network models typically have attractors corresponding to grid points such that parallel parsing is inherently unstable. Even in the absence of disambiguating information, such parsers will eventually abandon parallel activation of multiple parses; each symbol (or feature) will eventually be fully active or fully inactive.

This work extended previous GSC parsers in three key ways. Inspired by prior proposals (Cho et al., 2014; Hale and Smolensky, 2006; Le and Zuidema, 2015; McClelland and Elman, 1986; Pollack, 1990; Smolensky, 1990; Smolensky et al., 2014; Socher et al., 2012), we introduced the brick role representational system and applied it to continuous-time online incremental processing. Unlike the interactive activation and competition models (McClelland and Elman, 1986; McClelland and Rumelhart, 1981; Rumelhart and McClelland, 1982), our model provides a way of representing deeply nested tree structures, not just three layers of units, and works on distributed representation of symbols. Unlike deep neural networks of semantic composition —either recursive neural networks (Pollack, 1990; Socher et al., 2012) or convolutional neural 
networks (Le and Zuidema, 2015)) —our model processes a sentence incrementally in continuous time, one word at a time, considering multiple possibilities of local structures simultaneously.

Second, the previously proposed (comprehension) commitment policy (Cho et al., 2018, 2017) - in which all roles share the same commitment value - failed when the maximum sentence length increases. We proposed that the GSC parser should instead incorporate multiple commitment level parameters, one per each set of roles aligned diagonally. Given the $k$-th word of a sentence, the new policy scheme allows the model to maintain uncertainty in brick roles aligned with terminal brick roles from $(1,1)$ to $(1, k-1)$ and prevents it from developing strong commitment to some fillers in currently irrelevant brick roles aligned with terminal roles from $(1, k+1)$ to $\left(1, N_{\max }\right)$. In our simulation studies, for a given grammar, the GSC parser could parse every sentence well with a common commitment policy. In future work, we aim to implement an adaptive control mechanism that can discover good commitment policies from experience using reinforcement learning.

Third, we proposed a heuristic algorithm to train a GSC parser to approximate a PCFG. The simulation results suggest that this approach allows the GSC model to perform well when approximating simple PCFGs. We are currently investigating how this approach generalizes to more complex cases. Our preliminary results suggest that the algorithm has difficulty with grammars that generate a large number of parse trees, especially when they create a large amount of local ambiguity. In such challenging cases, trained models generated high-frequency parse trees spontaneously but had trouble generating low-frequency parse trees. Similarly, the trained model could parse high-frequency sentence types well but had trouble parsing low-frequency sentence types. Note that by re-training the model parameters $\mathbf{W}$ for parsing only, we could increase parsing accuracy significantly. This may provide support for an alternative approach, one that treats parsing and production separately. This provides another important direction for future work.

A key avenue for further development of this proposal is to relate its performance with human linguistic behaviors. There are several other models that provide baselines for comparison (Vosse and Kempen, 2000, 2009). Two interesting cases have been explored in previous work: the garden-path effect (Bever, 1970; Frazier and Rayner, 1982) and syntactically local coherence effects (Konieczny, 2005; Tabor et al., 2004). Previously, Cho et al. (2017) proposed an integrated GSC account of both effects: parsing failures can arise from suboptimal commitment policies. If the commitment level is too low, the model fails to encode information available from the present word input so later the model may build an analysis consistent with locally available information but inconsistent with distant past information (local coherence effect; e.g., "The coach smiled at the player tossed a frisbee by the team" [Tabor et al., 2004]). If the commitment level is too high, the model may choose one interpretation over the others too early, in which case it can select an interpretation which turns out to be inconsistent with later input (garden path effect; e.g., "The horse raced past the barn fell" [Bever, 1970]). However, these claims are based on the investigation of the model's behavior as it processes the minimal cases: two-word sentences. Those claims need to be tested on more realistic cases.

A second direction for future work is relating the proposed GSC model to structural probabilistic models (Hale, 2001; Levy, 2008; Levy et al., 2009). A deep understanding of the relationship will bridge between structural probabilistic models assuming discrete symbolic structures as their hypothesis space and neural 
network models that assume a continuous representation space. Cho et al. (2018) makes initial steps in this direction. We have argued here that the conjunctive blend state of a GSC model can be interpreted as a dynamic encoding of a probability distribution over complete parse trees. Our model provides a chance to test their proposal on more general cases. In particular, an interesting question is how information-theoretic complexity metrics (e.g., surprisal and entropy) can be interpreted in the context of GSC models.

By mathematically linking symbolic with sub-symbolic representations, static grammars with dynamical parsers, and discrete with continuous representations and processes, GSC provides a framework for developing proposals that integrate across levels of analysis and alternative cognitive architectures. The GSC model of online sentence comprehension proposes a neurally plausible, symbolically interpretable, and potentially scalable solution to core computational problems arising in online language processing.

\section{Acknowledgements}

Supported by NSF INSPIRE Grant BCS1344269 and the College of Literature, Science and Arts, University of Michigan. Thanks to Rick Lewis and Whit Tabor for helpful comments on this work.

\section{Appendix A Recursive construction of brick roles}

Smolensky and Legendre (2006a) present how to get vector encodings of recursive roles from a set of elementary role vectors by applying the tensor product operation recursively. The same idea can be applied to brick roles, although in the opposite direction. Let $\mathbf{p}^{(p)}$ be the vector encoding of a structural role $\mathrm{p}$, representing a position in a surface string. Let $\mathbf{0}$ and $\mathbf{1}$ be the vector encodings of the left and the right child of their parent position. Brick role $(l, p)$ is defined recursively as follows: $(l, p) \equiv \mathbf{r}^{(1, p)}=\mathbf{p}^{(p)}$ if $l=1$ and $(l, p) \equiv \mathbf{r}^{(l, p)}=\mathbf{r}^{(l-1, p)} \otimes \mathbf{0}+\mathbf{r}^{(l-1, p+1)} \otimes \mathbf{1}$ if $l$ is an integer greater than 1. For example, $(3,1)$ $\equiv \mathbf{r}^{(3,1)}=\mathbf{r}^{(2,1)} \otimes \mathbf{0}+\mathbf{r}^{(2,2)} \otimes \mathbf{1}=\left(\mathbf{r}^{(1,1)} \otimes \mathbf{0}+\mathbf{r}^{(1,2)} \otimes \mathbf{1}\right) \otimes \mathbf{0}+\left(\mathbf{r}^{(1,2)} \otimes \mathbf{0}+\mathbf{r}^{(1,3)} \otimes \mathbf{1}\right) \otimes \mathbf{1}=\mathbf{p}^{(1)} \otimes(\mathbf{0} \otimes \mathbf{0})+$ $\mathbf{p}^{(2)} \otimes(\mathbf{0} \otimes \mathbf{1}+\mathbf{1} \otimes \mathbf{0})+\mathbf{p}^{(3)} \otimes(\mathbf{1} \otimes \mathbf{1})$. For simplicity, in the present study, we assume every role vector is orthogonal to every other role vector and reserve the question of the effect of role similarity on processing dynamics for future studies.

\section{References}

Ackley, D. H., Hinton, G. E., and Sejnowski, T. J. (1985). A learning algorithm for Boltzmann machines. Cognitive Science, 9(1):147-169.

beim Graben, P. and Hutt, A. (2015). Detecting event-related recurrences by symbolic analysis: Applications to human language processing. Philosophical Transactions of the Royal Society A: Mathematical, Physical and Engineering Sciences, 373(2034):20140089.

Bever, T. G. (1970). The cognitive basis for linguistic structures. In Hayes, J. R., editor, Cognition and the Development of Language, pages 279-362. John Wiley, New York. 
Boston, M. F., Hale, J., Kliegl, R., Patil, U., and Vasishth, S. (2008). Parsing costs as predictors of reading difficulty: An evaluation using the Potsdam Sentence Corpus. Journal of Eye Movement Research, 2(1):112.

Boston, M. F., Hale, J. T., Vasishth, S., and Kliegl, R. (2011). Parallel processing and sentence comprehension difficulty. Language and Cognitive Processes, 26(3):301-349.

Chiang, T., Hwang, C., and Sheu, S. (1987). Diffusion for global optimization in \$\mathbb $\{\mathrm{R}\} \hat{\mathrm{n}} \$$. SIAM Journal on Control and Optimization, 25(3):737-753.

Cho, K., van Merrienboer, B., Bahdanau, D., and Bengio, Y. (2014). On the properties of neural machine translation: Encoder-decoder approaches. arXiv:1409.1259 [cs, stat].

Cho, P. W., Goldrick, M., Lewis, R. L., and Smolensky, P. (2018). Dynamic encoding of structural uncertainty in gradient symbols. In Proceedings of the 8th Workshop on Cognitive Modeling and Computational Linguistics (CMCL 2018), pages 19-28.

Cho, P. W., Goldrick, M., and Smolensky, P. (2017). Incremental parsing in a continuous dynamical system: Sentence processing in Gradient Symbolic Computation. Linguistics Vanguard, 3(1).

Chomsky, N. (1957). Syntactic structures. The Hague: Mouton.

Christiansen, M. H. and Chater, N. (2016). The Now-or-Never bottleneck: A fundamental constraint on language. Behavioral and Brain Sciences, 39:e62 (19 pages).

Cowan, N. (2001). The magical number 4 in short-term memory: A reconsideration of mental storage capacity. The Behavioral and Brain Sciences, 24(1):87-114; discussion 114-185.

Culbertson, J., Smolensky, P., and Wilson, C. (2013). Cognitive biases, linguistic universals, and constraintbased grammar learning. Topics in Cognitive Science, 5(3):392-424.

Demberg, V. and Keller, F. (2008). Data from eye-tracking corpora as evidence for theories of syntactic processing complexity. Cognition, 109(2):193-210.

Dillon, B., Andrews, C., Rotello, C. M., and Wagers, M. (2017). A new argument for co-active parses during language comprehension. Open Science Framework.

Ding, N., Melloni, L., Zhang, H., Tian, X., and Poeppel, D. (2016). Cortical tracking of hierarchical linguistic structures in connected speech. Nature Neuroscience, 19(1):158-164.

Dolan, C. P. (1989). Tensor Manipulation Networks: Connectionist and Symbolic Approaches to Comprehension, Learning, and Planning. PhD Thesis, University of California at Los Angeles, Los Angeles, CA, USA.

Dolan, C. P. and Smolensky, P. (1989). Tensor product production system: A modular architecture and representation. Connection Science, 1(1):53-68. 
Edelman, S. (2002). Constraining the neural representation of the visual world. Trends in Cognitive Sciences, 6(3):125-131.

Elman, J. L. (1990). Finding structure in time. Cognitive Science, 14(2):179-211.

Ferreira, F. and Henderson, J. M. (1991). Recovery from misanalyses of garden-path sentences. Journal of Memory and Language, 30(6):725-745.

Frank, S. and Thompson, R. (2012). Early effects of word surprisal on pupil size during reading. In Proceedings of the Cognitive Science Society, volume 34.

Frank, S. L., Bod, R., and Christiansen, M. H. (2012). How hierarchical is language use? Proceedings of the Royal Society of London B: Biological Sciences, page rspb20121741.

Frank, S. L., Otten, L. J., Galli, G., and Vigliocco, G. (2015). The ERP response to the amount of information conveyed by words in sentences. Brain and Language, 140:1-11.

Frazier, L. (1985). Syntactic complexity. In Dowty, D. R., Karttunen, L., and Zwicky, A. M., editors, Natural Language Processing: Psychological, Computational and Theoretical Perspectives, pages 129189. Cambridge University Press, Cambridge, UK.

Frazier, L. (1987). Sentence processing: A tutorial review. In Coltheart, M., editor, Attention and Performance XII: The Psychology of Reading, pages 559-586. Lawrence Erlbaum Associates.

Frazier, L. and Rayner, K. (1982). Making and correcting errors during sentence comprehension: Eye movements in the analysis of structurally ambiguous sentences. Cognitive Psychology, 14(2):178-210.

Gayler, R. W. (2003). Vector symbolic architectures answer Jackendoff's challenges for cognitive neuroscience. In Slezak, P., editor, Proceedings of the ICCS/ASCS International Conference on Cognitive Science, pages 133-138, Sydney, Australia: University of New South Wales.

Gibson, E. and Thomas, J. (1999). Memory limitations and structural forgetting: The perception of complex ungrammatical sentences as grammatical. Language and Cognitive Processes, 14(3):225-248.

Hale, J. (2001). A probabilistic Earley parser as a psycholinguistic model. In Proceedings of the Second Meeting of the North American Chapter of the Association for Computational Linguistics on Language Technologies, NAACL '01, pages 1-8, Stroudsburg, PA, USA. Association for Computational Linguistics.

Hale, J. (2003). The information conveyed by words in sentences. Journal of Psycholinguistic Research, 32(2):101-123.

Hale, J. (2016). Information-theoretical complexity metrics. Language and Linguistics Compass, 10(9):397-412.

Hale, J. and Smolensky, P. (2006). Harmonic Grammars and harmonic parsers for formal languages. In Smolensky, P. and Legendre, G., editors, The Harmonic Mind: From Neural Computation to OptimalityTheoretic Grammar. Volume I: Cognitive Architecture, pages 393-416. The MIT Press. 
Hayes, B. and Wilson, C. (2008). A maximum entropy model of phonotactics and phonotactic learning. Linguistic Inquiry, 39(3):379-440.

Henderson, J. M., Choi, W., Lowder, M. W., and Ferreira, F. (2016). Language structure in the brain: A fixation-related fMRI study of syntactic surprisal in reading. NeuroImage, 132:293-300.

Hochreiter, S. and Schmidhuber, J. (1997). Long short-term memory. Neural Computation, 9(8):1735-1780.

Hockett, C. F. (1960). The origin of speech. Scientific American, 203:89-96.

Hopfield, J. J. (1984). Neurons with graded response have collective computational properties like those of two-state neurons. Proceedings of the National Academy of Sciences, 81(10):3088-3092.

Hutt, A. and beim Graben, P. (2017). Sequences by metastable attractors: Interweaving dynamical systems and experimental data. Frontiers in Applied Mathematics and Statistics, 3:11.

Jurafsky, D. (1996). A probabilistic model of lexical and syntactic access and disambiguation. Cognitive Science, 20(2):137-194.

Kempen, G. (2014). Prolegomena to a neurocomputational architecture for human grammatical encoding and decoding. Neuroinformatics, 12(1):111-142.

Kempen, G., Olsthoorn, N., and Sprenger, S. (2012). Grammatical workspace sharing during language production and language comprehension: Evidence from grammatical multitasking. Language and Cognitive Processes, 27(3):345-380.

King, J. and Just, M. A. (1991). Individual differences in syntactic processing: The role of working memory. Journal of Memory and Language, 30(5):580-602.

Konieczny, L. (2005). The psychological reality of local coherences in sentence processing. In Proceedings of the 27th Annual Conference of the Cognitive Science Society, pages 1178-1183.

Le, P. and Zuidema, W. (2015). The forest convolutional network: Compositional distributional semantics with a neural chart and without binarization. In Proceedings of the 2015 Conference on Empirical Methods in Natural Language Processing, pages 1155-1164.

LeCun, Y., Bottou, L., Bengio, Y., and Haffner, P. (1998). Gradient-based learning applied to document recognition. Proceedings of the IEEE, 86(11):2278-2324.

Legendre, G., Miyata, Y., and Smolensky, P. (1990). Harmonic grammar - A formal multi-level connectionist theory of linguistic well-formedness: Theoretical foundations. Technical Report CU-CS-465-90, Department of Computer Science, University of Colorado Boulder.

Levy, R. (2008). Expectation-based syntactic comprehension. Cognition, 106(3):1126-1177. 
Levy, R., Bicknell, K., Slattery, T., and Rayner, K. (2009). Eye movement evidence that readers maintain and act on uncertainty about past linguistic input. Proceedings of the National Academy of Sciences, 106(50):21086-21090.

Lewis, R. L. and Vasishth, S. (2005). An activation-based model of sentence processing as skilled memory retrieval. Cognitive Science, 29(3):375-419.

Lowder, M. W., Choi, W., Ferreira, F., and Henderson, J. M. (2018). Lexical predictability during natural reading: Effects of surprisal and entropy reduction. Cognitive Science, 42(S4):1166-1183.

MacDonald, M. C., Pearlmutter, N. J., and Seidenberg, M. S. (1994). The lexical nature of syntactic ambiguity resolution. Psychological Review, 101(4):676-703.

Marr, D. (1982). Vision: A Computational Investigation into the Human Representation and Processing of Visual Information. MIT Press.

McClelland, J. L. and Elman, J. L. (1986). The TRACE model of speech perception. Cognitive Psychology, 18(1):1-86.

McClelland, J. L. and Rumelhart, D. E. (1981). An interactive activation model of context effects in letter perception: I. An account of basic findings. Psychological Review, 88(5):375-407.

McRae, K., Spivey-Knowlton, M. J., and Tanenhaus, M. K. (1998). Modeling the influence of thematic fit (and other constraints) in on-line sentence comprehension. Journal of Memory and Language, 38(3):283312.

Menenti, L., Gierhan, S. M. E., Segaert, K., and Hagoort, P. (2011). Shared language: Overlap and segregation of the neuronal infrastructure for speaking and listening revealed by functional mri. Psychological Science, 22(9):1173-1182.

Miller, G. A. (1956). The magical number seven, plus or minus two: Some limits on our capacity for processing information. Psychological Review, 63(2):81-97.

Movellan, J. R. (1998). A learning theorem for networks at detailed stochastic equilibrium. Neural Computation, 10(5):1157-1178.

Movellan, J. R. and McClelland, J. L. (1993). Learning continuous probability distributions with symmetric diffusion networks. Cognitive Science, 17(4):463-496.

Nelson, M. J., Karoui, I. E., Giber, K., Yang, X., Cohen, L., Koopman, H., Cash, S. S., Naccache, L., Hale, J. T., Pallier, C., and Dehaene, S. (2017). Neurophysiological dynamics of phrase-structure building during sentence processing. Proceedings of the National Academy of Sciences, 114(18):E3669-E3678.

Newell, A. (1980). Physical symbol systems. Cognitive Science, 4(2):135-183.

Pickering, M. J. and Ferreira, V. S. (2008). Structural priming: A critical review. Psychological Bulletin, 134(3):427-459. 
Pinker, S. (1994). The Language Instinct: How the Mind Creates Language. William Morrow \& Company, New York.

Plate, T. A. (1995). Holographic reduced representations. IEEE Transactions on Neural networks, 6(3):623641.

Pollack, J. B. (1990). Recursive distributed representations. Artificial Intelligence, 46(1-2):77-105.

Rasmussen, N. E. and Schuler, W. (2018). Left-corner parsing with distributed associative memory produces surprisal and locality effects. Cognitive science, 42:1009-1042.

Roark, B., Bachrach, A., Cardenas, C., and Pallier, C. (2009). Deriving lexical and syntactic expectationbased measures for psycholinguistic modeling via incremental top-down parsing. In Proceedings of the 2009 Conference on Empirical Methods in Natural Language Processing: Volume 1-Volume 1, pages 324-333. Association for Computational Linguistics.

Rumelhart, D. E. and McClelland, J. L. (1982). An interactive activation model of context effects in letter perception: II. The contextual enhancement effect and some tests and extensions of the model. Psychological Review, 89(1):60-94.

Segaert, K., Menenti, L., Weber, K., Petersson, K. M., and Hagoort, P. (2012). Shared syntax in language production and language comprehension—an fMRI study. Cerebral Cortex, 22(7):1662-1670.

Smith, G., Franck, J., and Tabor, W. (2018). A self-organizing approach to subject-verb number agreement. Cognitive Science, 42:1043-1074.

Smith, N. J. and Levy, R. (2013). The effect of word predictability on reading time is logarithmic. Cognition, 128(3):302-319.

Smolensky, P. (1986). Neural and conceptual interpretation of PDP models. In Parallel Distributed Processing: Explorations in the Microstructure of Cognition, Vol. 2: Psychological and Biological Models, pages 390-431. MIT Press, Cambridge, MA.

Smolensky, P. (1990). Tensor product variable binding and the representation of symbolic structures in connectionist systems. Artificial Intelligence, 46(1):159-216.

Smolensky, P. (1993). Harmonic grammars for formal languages. In Hanson, S. J., Cowan, J. D., and Giles, C. L., editors, Advances in Neural Information Processing Systems 5, pages 847-854. Morgan-Kaufmann.

Smolensky, P. (2006). Tensor product representations: Formal foundations. P. Smolensky \& G. Legendre, The harmonic mind: From neural computation to optimality-theoretic grammar, 1:271-344.

Smolensky, P., Goldrick, M., and Mathis, D. (2014). Optimization and quantization in gradient symbol systems: A framework for integrating the continuous and the discrete in cognition. Cognitive Science, 38(6):1102-1138. 
Smolensky, P. and Legendre, G., editors (2006a). The Harmonic Mind: From Neural Computation to Optimality-Theoretic Grammar. Volume 1: Cognitive Architecture. The MIT Press, Cambridge, MA.

Smolensky, P. and Legendre, G., editors (2006b). The Harmonic Mind: From Neural Computation to Optimality-Theoretic Grammar. Volume 2: Linguistic and Philosophical Implications. The MIT Press, Cambridge, MA.

Smolensky, P. and Tesar, B. (2006). Symbolic computation with activation patterns. P. Smolensky \& G. Legendre, The harmonic mind: From neural computation to optimality-theoretic grammar, 1:235-270.

Socher, R., Huval, B., Manning, C. D., and Ng, A. Y. (2012). Semantic compositionality through recursive matrix-vector spaces. In Proceedings of the 2012 Joint Conference on Empirical Methods in Natural Language Processing and Computational Natural Language Learning, EMNLP-CoNLL '12, pages 1201-1211, Stroudsburg, PA, USA. Association for Computational Linguistics.

Stevenson, S. (1994). Competition and recency in a hybrid network model of syntactic disambiguation. Journal of Psycholinguistic Research, 23(4):295-322.

Tabor, W., Galantucci, B., and Richardson, D. (2004). Effects of merely local syntactic coherence on sentence processing. Journal of Memory and Language, 50(4):355-370.

Tabor, W. and Hutchins, S. (2004). Evidence for self-organized sentence processing: Digging-in effects. Journal of Experimental Psychology: Learning, Memory, and Cognition, 30(2):431-450.

Traxler, M. J., Pickering, M. J., and Clifton Jr., C. (1998). Adjunct attachment is not a form of lexical ambiguity resolution. Journal of Memory and Language, 39(4):558-592.

Tupper, P., Smolensky, P., and Cho, P. W. (2014). Discrete symbolic optimization and Boltzmann sampling by continuous neural dynamics: Gradient Symbolic Computation. arXiv:1409.1259 [cs].

van Gompel, R. P. G., Pickering, M. J., and Traxler, M. J. (2001). Reanalysis in sentence processing: Evidence against current constraint-based and two-stage models. Journal of Memory and Language, 45(2):225-258.

Van Schijndel, M. and Linzen, T. (2018). Modeling garden path effects without explicit hierarchical syntax. In Proceedings of the 40th Annual Meeting of the Cognitive Science Society.

von der Malsburg, C. (1999). The what and why of binding: The modeler's perspective. Neuron, 24(1):95104.

Vosse, T. and Kempen, G. (2000). Syntactic structure assembly in human parsing: A computational model based on competitive inhibition and a lexicalist grammar. Cognition, 75(2):105-143.

Vosse, T. and Kempen, G. (2009). The Unification Space implemented as a localist neural net: Predictions and error-tolerance in a constraint-based parser. Cognitive Neurodynamics, 3(4):331-346. 
Willems, R. M., Frank, S. L., Nijhof, A. D., Hagoort, P., and van den Bosch, A. (2016). Prediction during natural language comprehension. Cerebral Cortex, 26(6):2506-2516. 